\title{
Tightening the Lieb-Robinson Bound in Locally Interacting Systems
}

\author{
Zhiyuan Wang $\oplus^{1,2,{ }^{*}}$ and Kaden R.A. Hazzard ${ }^{1,2}$ \\ ${ }^{1}$ Department of Physics and Astronomy, Rice University, Houston, Texas 77005, USA \\ ${ }^{2}$ Rice Center for Quantum Materials, Rice University, Houston, Texas 77005, USA
}

(Received 12 August 2019; revised 3 January 2020; accepted 22 July 2020; published 3 September 2020)

\begin{abstract}
The Lieb-Robinson (LR) bound rigorously shows that in quantum systems with short-range interactions, the maximum amount of information that travels beyond an effective "light cone" decays exponentially with distance from the light-cone front, which expands at finite velocity. Despite being a fundamental result, existing bounds are often extremely loose, limiting their applications. We introduce a method that dramatically and qualitatively improves LR bounds in models with finite-range interactions. Most prominently, in systems with a large local Hilbert space dimension $D$, our method gives a LR velocity that grows much slower than previous bounds with $D$ as $D \rightarrow \infty$. For example, in the Heisenberg model with spin $S$, we find $v \leq$ const. compared to the previous $v \propto S$, which diverges at large $S$, and in multiorbital Hubbard models with $N$ orbitals, we find $v \propto \sqrt{N}$ instead of previous $v \propto N$, and similarly in the $N$ state truncated Bose-Hubbard model and Wen's quantum rotor model. Our bounds also scale qualitatively better in some systems when the spatial dimension or certain model parameters become large, for example in the $d$-dimensional quantum Ising model and perturbed toric code models. Even in spin-1/2 Ising and Fermi-Hubbard models, our method improves the LR velocity by an order of magnitude with typical model parameters, and significantly improves the LR bound at large distance and early time.
\end{abstract}

DOI: 10.1103/PRXQuantum.1.010303

\section{INTRODUCTION}

The Lieb-Robinson (LR) bound [1,2] has revealed how locality shapes and constrains quantum matter. It says that in short-range (exponentially decaying) interacting quantum systems on a lattice, the influence of any local perturbation is restricted to an effective light cone expanding at a finite speed, apart from an exponentially decaying tail outside the light cone. It has direct implications for many-body quantum dynamics, as it has been used to understand the timescales necessary to generate correlation and entanglement [3-6], to transfer information in a quantum channel [7,8], and to prethermalize a system [9-12]. It has also found applications to equilibrium properties, for example in proving that correlations decay exponentially in a gapped ground state (the exponential clustering theorem [13-19]), that systems with half integer spin per unit cell are necessarily gapless (the Lieb-Schultz-MattisHastings theorem [20-22]), the area law for entanglement entropy in ground states of gapped systems [23-25],

*zhiyuan.wang@rice.edu

Published by the American Physical Society under the terms of the Creative Commons Attribution 4.0 International license. Further distribution of this work must maintain attribution to the author(s) and the published article's title, journal citation, and DOI. the stability of topological order [26-29], and the formal proof for the quantization of the Hall conductance as integer multiples of $e^{2} / h$ [30]. It is also the theoretical basis for analyzing the accuracy of some numerical algorithms [31-36]. It has been generalized to systems with long-range power-law decaying interaction [14,3646], to $n$-partite connected correlation functions [47], and to dynamics with Markovian dissipation [48-50] as well. Furthermore, recent developments in experimental technology of atomic, molecular, and optical physics have enabled experimentalists to observe the effective light cone and measure the LR speed $[38,51,52]$.

Despite the LR bounds' fundamental consequences and efforts to tighten them $[14,15,49,53-55]$, the bounds are often extremely loose [except some special bounds on freeparticle systems [56-60] ], severely limiting their applicability. To see this, note that in locally interacting systems, previous LR bounds are typically of the form

$$
\left\|\left[\hat{A}_{X}(t), \hat{B}_{Y}(0)\right]\right\| \leq C e^{-s_{X Y} / \xi}\left(e^{v|t| / \xi}-1\right),
$$

where $\hat{A}_{X}, \hat{B}_{Y}$ are local operators supported on regions $X, Y$, respectively, $\hat{A}_{X}(t)=e^{i \hat{H} t} \hat{A}_{X} e^{-i \hat{H} t}, s_{X Y}$ is the minimal graph-theoretical distance between points in $X$ and $Y$, and $v$ and $\xi$ are constants that only depend on the Hamiltonian $\hat{H}$ (and the lattice structure). There are two qualitative ways in which these bounds are loose. First, 
and arguably most importantly, the LR speeds $v$ appearing in existing bounds are typically much larger than the actual speed of information propagation. For example, in the onedimensional (1D) transverse-field Ising model (TFIM) at critical point, the previous best LR speed is $8 e J \approx 21.7 \mathrm{~J}$ while the actual speed determined from exact solution is $2 J$. Previous bounds on $v$ can even become infinitely loose in certain limits: for example in the TFIM at large $J$, previous bounds grow $\propto J$; in the spin- $S$ Heisenberg model, previous bounds grow $\propto S$; in the classical limit of the $\mathrm{SU}(N)$ Fermi-Hubbard (FH) model, previous bounds grow $\propto N$, while in all these cases the true LR speed remains finite. Previous bounds are also qualitatively loose at short time and large distance: as $t \rightarrow 0$, perturbative arguments show that the lhs of Eq. (1) grows like $O\left(t^{c_{X Y}}\right)$, where $c$ is a constant, much slower than the $O(t)$ in the rhs of Eq. (1); similarly, as $s_{X Y} \rightarrow \infty$, the lhs actually decays faster than exponential. A simple example is noninteracting systems, where the lhs decays at large distance like $O\left(e^{-s_{X Y} \ln s_{X Y}}\right)$.

In this paper, we focus on locally interacting systems (i.e. systems with finite-range interactions), and introduce a method that qualitatively tightens the LR bounds in all the aforementioned aspects. The key new ingredient in our method is the commutativity graph, which is a graphical tool that helps us taking advantage of the commutativity structure of the Hamiltonian terms, a feature that has been ignored by previous methods (except in a special model in Ref. [53]). By applying the Heisenberg equation and triangle inequality, we obtain a system of linear integral inequalities, which only involve the unequal time commutator of the Hamiltonian terms. We then find a system of linear differential equations whose solution gives an upper bound for any solution to the integral inequalities. These differential equations can be naturally understood as the information flow equation on the commutativity graph.

If the quantitative tightness is the sole criterion for applying the LR bound, one should solve these equations either analytically, or numerically if necessary, which can be done quickly and straightforwardly for systems with many thousands of sites [since the number of variables is proportional to the size of the system, see Eq. (10)]. In translationally invariant systems, the solution to the system of linear differential equations can be reduced to an integral by Fourier transformation. For example, in the $d$-dimensional TFIM, our LR bound for $\left[\hat{\sigma}_{\vec{r}}^{x}(t), \hat{\sigma}_{\overrightarrow{0}}^{z}(0)\right]$ is

$$
\left\|\left[\hat{\sigma}_{\vec{r}}^{x}(t), \hat{\sigma}_{\overrightarrow{0}}^{z}(0)\right]\right\| \leq \int_{-\pi}^{\pi} \cosh [\omega(\vec{k}) t] e^{i \vec{k} \cdot \vec{r}} \frac{d^{d} k}{(2 \pi)^{d}},
$$

where $\omega(k)$ corresponds to eigenvalue of the linear operator encoding the differential equations, which can be found by diagonalizing a $(d+1) \times(d+1)$ matrix for each $k$.
The bound for other operators takes a similar form. Based on the Fourier integral representation, we provide a general method to analyze the asymptotic behaviors of the solutions - which shows that the small $t$ and large $r$ behavior is qualitatively tighter than prior bounds, and are often the tightest possible - and derive an explicit formula to extract the LR speed. The LR speed obtained by this method vastly improves previous ones, as summarized in Tables I and II.

As we see, there are three broad scenarios where the LR velocity appearing in our bounds is qualitatively tighter: (1) when the number of local degrees of freedom becomes large, e.g., the large-S limit in TFIM, Heisenberg $X Y Z$ models, and Wen's quantum rotor model; the BoseHubbard (BH) model truncated to allow at most $N$ particles per site, and the large- $N$ limit in the $\mathrm{SU}(N) \mathrm{FH}$ model, (2) when the parameters of a set of commuting operators becomes large (e.g., large- $J$ limit in TFIM, perturbed toric code model), and (3) TFIM and Wen's quantum rotor model in large spatial dimension. The bounds we present shed light onto these important limits, which have previously resisted bounds with the qualitatively correct scaling. They also have important physical implications, for example in exactly solvable models with topological order [e.g., toric code [61] and string net models [62]], perturbed by an arbitrary bounded, local perturbation. Our results show that their LR velocity vanishes as the strength of the perturbation approaches zero, in contrast to prior bounds, which retained a finite LR velocity in this limit. Even in situations with low spatial dimension and small local degrees of freedom, e.g., at $J=h$ in two-dimensional (2D) TFIM or

TABLE I. Comparison between previous LR speeds and those introduced in this paper, in the case of 2D spin-1/2 TFIM, 1D SU(2) FH model, and perturbed toric code (PTC) model [61] with onsite $h \sum_{j} \hat{\sigma}_{j}^{x}$ perturbation. The LR speed $v_{\mathrm{LR}}$ of this paper is upper bounded by the minimum of all the expressions in the different rows of each model. For each column divided into two subcolumns, the first subcolumn shows analytic expressions, and the second subcolumn shows their approximate numerical values. (For those bounds of the FH model depending on $U / J$ we have chosen a representative point). The constant $X_{y}$ is defined as the solution to the equation $x \operatorname{arcsinh}(x)=\sqrt{x^{2}+1}+y \cdot Z_{U / J}$ is defined in Eq. (81) and plotted in Fig. 5.

\begin{tabular}{lcccr}
\hline \hline Model & \multicolumn{2}{c}{$v_{\text {LR }}$ (this paper) } & \multicolumn{2}{c}{$v_{\text {LR }}$ (Ref. [2]) } \\
\hline 2D TFIM & $2 X_{0} \sqrt{2 J h}$ & $4.27 \sqrt{J h}$ & $16 e J$ & $43.5 J$ \\
& $8 X_{1 / 2} J$ & $15.1 J$ & & \\
& $8 X_{0} h$ & $12.1 h$ & & \\
1 D FH & $2 X_{3 U / 4 J} J$ & $4.14 J_{(U=J)}$ & $16 e J$ & $43.5 J$ \\
& $8 X_{0} J$ & $12.1 J$ & & \\
& $Z_{U / J} J$ & $7.05 J_{(U=5 J)}$ & & \\
PTC & $8 X_{1 / 2} h$ & $15.1 h$ & $32 e$ & 87.0 \\
& $2 X_{0} \sqrt{2 h}$ & $4.27 \sqrt{h}$ & & \\
& $8 X_{0}$ & 12.1 & & \\
\hline \hline
\end{tabular}


TABLE II. Summary of qualitative improvements of $v_{\text {LR }}$ in this paper for models with large onsite Hilbert spaces or large spatial dimensions: $S$ indicates the size of spins in spin models, $N$ the maximal number of particles on a single site in Hubbard models, and $d$ the spatial dimension. In Wen's rotor model, we only list the result in the most interesting limit $S \rightarrow \infty, J \ll g$, where the previous best methods from Refs. [2] and [53] are infinitely looser than our result.

\begin{tabular}{lcc}
\hline \hline Model & $v_{\text {LR }}$ (this paper) & $v_{\text {LR }}$ (Ref. [2]) \\
\hline Large- $d$ TFIM & $\propto \sqrt{d}$ & $\propto d$ \\
Large- $S$ (spin) TFIM & $\propto \sqrt{S}$ & $\propto S^{2}$ \\
Large- $N$ SU( $N$ ) FH & $\propto \sqrt{N}$ & $\propto N$ \\
Spin- $S$ Heisenberg & const. & $\propto S$ \\
Wen's rotor model, & $\propto \sqrt{d g J}$ & $\propto \sqrt{J g} d S$ (Ref. [53]) \\
$S \rightarrow \infty$ and $J \ll g$ & $\propto \sqrt{N}$ & $\propto d g$ (Ref. [2]) \\
$N$-state truncated BH & $\propto \sqrt{N}$ & $\propto N$ \\
\hline \hline
\end{tabular}

$U=J$ in 1D FH, our bound represents more than a tenfold improvement.

Although expressions such as Eq. (2) provide the tightest bounds in this paper, we can find simple analytic bounds while compromising the tightness only marginally. We show that

$$
\left\|\left[\hat{A}_{X}(t), \hat{B}_{Y}(0)\right]\right\| \leq C\left(\frac{u|t|}{d_{X Y}}\right)^{d_{X Y}} .
$$

We note that this bound applies even to systems lacking translational invariance (under mild realistic requirements on the Hamiltonian to be introduced later). Here $d_{X Y}$ is the distance between operators $\hat{A}_{X}(t), \hat{B}_{Y}(0)$ on the commutativity graph $\left(d_{X Y}\right.$ grows linearly with the distance between the two operators in real space), $C$ is a constant, and $u$ is related to the LR speed given in Tables I, II (their precise relation is given in Secs. IV and V). This bound also tightens previous ones in the two aspects mentioned above, and its asymptotic behavior at small time and large distance is often tightest possible. For example, the small- $t$ exponent is the same as the exact one obtained by perturbative arguments and the large- $x$ exponent is saturated by some free-particle systems.

The tighter LR bounds also improve several important results that rely on it. We mention two here. The first, which we explicitly derive in this paper, is that our LR bounds give rise to a tighter bound for the groundstate correlation length in systems with a spectral gap. For example, when applied to the 1D TFIM, our bound gives $\xi \propto[\ln (J / h)]^{-1}$ in the limit $J / h \rightarrow \infty$, in agreement with the exact solution, while the previous best bound approaches a constant; in the spin- $S$ Heisenberg model, when $S \rightarrow \infty$, our bound gives $\xi \leq c_{1}$ (a constant), while the previous bound $\xi \leq c_{2}(S+1)$ diverges linearly in $S$. As a second example, the improvement of the bound also enables further applications where quantitative accuracy is the key to obtaining helpful results, for example in upper bounding the error of several numerical algorithms [31-33,35,36]. Previous LR bounds give extremely loose numerical error bounds, which renders the error bound practically useless in typical situations where only modest system sizes can be simulated with current computational capabilities. We expect the tighter LR bounds to give practically useful numerical error bounds for reasonably small system sizes.

Our paper is organized as follows. In Sec. II we outline the general method to upper bound the unequal time commutator by the solution of a set of first-order linear differential equations. In Sec. III we focus on translation invariant systems, where one can obtain Fourier integral solutions to the differential equations and obtain the bound in Eq. (2). We also derive an explicit formula for the LR speed by studying the analytic properties of the Fourier integrals. In Sec. IV we derive a power series solution to the aforementioned differential equations in an arbitrary graph, from which we prove the LR bound in Eq. (3). In Sec. V we give examples of our LR bounds in some paradigmatic models (TFIM, spin- $S$ Heisenberg, $N$-state truncated $\mathrm{BH}, \mathrm{SU}(N)$ $\mathrm{FH}$, and Wen's quantum rotor model). In Sec. VI we introduce a special treatment that can be used to obtain a tighter bound when some of the parameters in the Hamiltonian become large. In Sec. VII we show how our LR bounds give a better bound on the ground-state correlation length in systems with a spectral gap. In Sec. VIII we summarize our methods and results, and discuss their potential applications along with some future directions. In Appendix A we briefly introduce a more general method that unifies some of the seemingly different approaches in the main text.

\section{GENERAL METHOD: INFORMATION PROPAGATION ON COMMUTATIVITY GRAPHS}

There are two major reasons why previous LR bounds are loose. First, the previous methods do not make use of the details of the Hamiltonian. For example, the derivations in Ref. [14] only makes use of how the operator norms of the interaction terms decay with interaction range, completely ignoring all other properties such as commutativity. Therefore, the previous results actually bound the "worst case" Hamiltonian that satisfies the finite-range condition. Second, previous derivations use the integral inequalities iteratively, leading to a sum of products of terms along different paths. Since this sum over paths is difficult to calculate analytically, one has to use the triangle inequality many times to get a simple analytic expression, which makes the resulting bound looser.

In the following, we derive our LR bounds using a method that avoids these two difficulties. Motivated by the 
LR bound of a special model studied in Ref. [53] in the context of topological matter, we make use of the details of the Hamiltonian by carefully exploiting the commutation relations of the terms in the Hamiltonian. To facilitate this procedure, we introduce a graphical tool, which we call the commutativity graph, that helps visualize the commutation relations of Hamiltonian terms, and is introduced in Sec. II A. In Sec. II B we use the Heisenberg equation and triangle inequality to upper bound the unequal time commutator and arrive at a similar integral inequality as previous LR bounds. However, instead of using it iteratively, we find a set of differential equations whose solution naturally provides an upper bound for any quantities satisfying the integral inequalities. A simple analytic bound for solutions to these differential equations is discussed in the next section, using a simpler method than the typical sum over paths in the previous approaches, and which produces much tighter bounds.

\section{A. Commutativity graph}

We begin by introducing a useful graphical tool, namely the commutativity graph, to represent the Hamiltonian of locally interacting systems. Consider a locally interacting quantum system in arbitrary dimension with an arbitrary lattice structure, or on an arbitrary graph. The Hamiltonian $\hat{H}$ can in general be written as

$$
\hat{H}=\sum_{j} h_{j} \hat{\gamma}_{j}
$$

where $\hat{\gamma}_{j}$ are local Hermitian operators with unit norm $\left\|\hat{\gamma}_{j}\right\|=1$, and $h_{j}$ are constant parameters.

The commutativity graph $G$ of the Hamiltonian $\hat{H}$ is constructed as follows. Each local operator $\hat{\gamma}_{i}$ is represented by a vertex $i$, the parameter $h_{i}$ is attached to vertex $i$, and we link two vertices $i, j$ by an edge $\langle i, j\rangle$ if and only if the corresponding terms do not commute $\left[\hat{\gamma}_{i}, \hat{\gamma}_{j}\right] \neq 0$. The resulting graph necessarily reflects the locality, since local operators acting on nonoverlapping spatial regions must commute, so there is no edge between their representative vertices. Some simple examples of commutativity graphs are shown in Fig. 1. Notice that the same Hamiltonian may have different decompositions in the form of Eq. (4) and, therefore, different commutativity graphs, due to the freedom in how to partition terms of $\hat{H}$. Nevertheless, for convenience we simply speak of "the commutativity graph of $\hat{H}$ " when a certain decomposition is implicitly assumed. We discuss how to choose the decomposition at the end of Sec. II B. In the following we derive a LR bound based on a differential equation on the commutativity graph of $\hat{H}$.

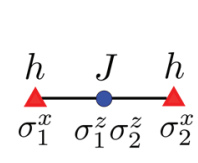

(a)

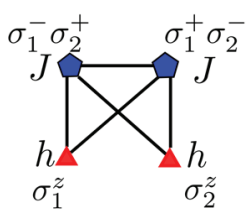

(b)

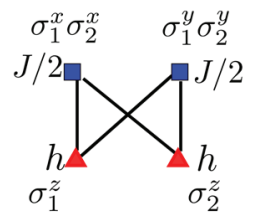

(c)
FIG. 1. Examples of commutativity graphs. (a) Commutativity graph of $\hat{H}_{1}=J \hat{\sigma}_{1}^{z} \hat{\sigma}_{2}^{z}+h\left(\hat{\sigma}_{1}^{x}+\hat{\sigma}_{2}^{x}\right)$. (b) Commutativity graph of $\hat{H}_{2}=J\left(\hat{\sigma}_{1}^{-} \hat{\sigma}_{2}^{+}+\hat{\sigma}_{1}^{+} \hat{\sigma}_{2}^{-}\right)+h\left(\hat{\sigma}_{1}^{z}+\hat{\sigma}_{2}^{z}\right)$. (c) Commutativity graph of $\hat{H}_{2}=J / 2\left(\hat{\sigma}_{1}^{x} \hat{\sigma}_{2}^{x}+\hat{\sigma}_{1}^{y} \hat{\sigma}_{2}^{y}\right)+h\left(\hat{\sigma}_{1}^{z}+\hat{\sigma}_{2}^{z}\right)$.

\section{B. Upper bound for $\left\|\left[\hat{\gamma}_{i}(t), \hat{B}(0)\right]\right\|$}

The Heisenberg equation for the operator $\hat{\gamma}_{i}(t)=$ $e^{i \hat{H} t} \hat{\gamma}_{i} e^{-i \hat{H} t}$ is

$$
i \dot{\hat{\gamma}}_{i}(t)=\left[\hat{\gamma}_{i}(t), \hat{H}\right]=\sum_{j:\langle i j\rangle \in G} h_{j}\left[\hat{\gamma}_{i}(t), \hat{\gamma}_{j}(t)\right]
$$

where the dot on $\hat{\gamma}_{i}(t)$ means time derivative, and the graph geometry provides a natural way to label the summation.

The general task of this paper is to find an upper bound for unequal time commutators $[\hat{A}(t), \hat{B}(0)]$ of local operators $\hat{A}, \hat{B}$, i.e., operators with finite support. Let us first consider the special case when $\hat{A}=\hat{\gamma}_{i}$ is a term of the Hamiltonian. In this case, we are interested in the unequal time commutator $\hat{\gamma}_{i}^{B}(t)=\left[\hat{\gamma}_{i}(t), \hat{B}(0)\right]$, for an arbitrary local operator $\hat{B}$. Using Eq. (5) and the Jacobi identity, we have the evolution equation for $\hat{\gamma}_{i}^{B}(t)$

$$
i \dot{\hat{\gamma}}_{i}^{B}(t)=\sum_{j:\langle i j\rangle \in G} h_{j}\left(\left[\hat{\gamma}_{i}^{B}(t), \hat{\gamma}_{j}(t)\right]+\left[\hat{\gamma}_{i}(t), \hat{\gamma}_{j}^{B}(t)\right]\right)
$$

Substituting $\hat{\gamma}_{i}^{B}(t)=\hat{U}(t) \hat{\tau}_{i}^{B}(t) \hat{U}^{\dagger}(t)$ where $\hat{U}(t)$ is the unitary operator satisfying $\hat{U}(0)=1$ and $i \dot{\hat{U}}(t)=-\sum_{j:\langle i j\rangle \in G}$ $h_{j} \hat{\gamma}_{j}(t) \hat{U}(t)$ into Eq. (6), we have

$$
i \dot{\hat{\tau}}_{i}^{B}(t)=\hat{U}^{\dagger}(t) \sum_{j:\langle i j\rangle \in G} h_{j}\left[\hat{\gamma}_{i}(t), \hat{\gamma}_{j}^{B}(t)\right] \hat{U}(t) .
$$

Now we bound the time dependence of the operator norm of $\hat{\gamma}_{i}^{B}(t)$, the fundamental object controlled by LR bounds. Since $\hat{\gamma}_{i}^{B}$ and $\hat{\tau}_{i}^{B}$ are related by unitary transforms, $\left\|\hat{\gamma}_{i}^{B}(t)\right\|=\left\|\hat{\tau}_{i}^{B}(t)\right\|$ (recall that $\left\|\hat{U} \hat{A} \hat{V}^{\dagger}\right\|=\|\hat{A}\|$ for unitary operators $\hat{U}, \hat{V})$. Using this and applying the basic inequalities $\|\hat{A}+\hat{B}\| \leq\|\hat{A}\|+\|\hat{B}\|$ and $\|\hat{A} \cdot \hat{B}\| \leq\|\hat{A}\| \cdot\|\hat{B}\|$, we 
obtain

$$
\begin{aligned}
& \left\|\hat{\gamma}_{i}^{B}(t)\right\|-\left\|\hat{\gamma}_{i}^{B}(0)\right\|=\left\|\hat{\tau}_{i}^{B}(t)\right\|-\left\|\hat{\tau}_{i}^{B}(0)\right\| \\
& \quad \leq \int_{0}^{t}\left\|\dot{\hat{\tau}}_{i}^{B}\left(t^{\prime}\right)\right\| d t^{\prime} \\
& \quad \leq \sum_{j:\langle i j\rangle \in G}\left|h_{j}\right| \int_{0}^{t}\left\|\left[\hat{\gamma}_{j}^{B}\left(t^{\prime}\right), \hat{\gamma}_{i}\left(t^{\prime}\right)\right]\right\| d t^{\prime} \\
& \quad \leq 2 \sum_{j:\langle i j\rangle \in G}\left|h_{j}\right| \int_{0}^{t}\left\|\hat{\gamma}_{j}^{B}\left(t^{\prime}\right)\right\| d t^{\prime},
\end{aligned}
$$

where in the last line we use $\left\|\hat{\gamma}_{i}\left(t^{\prime}\right)\right\|=\left\|\hat{\gamma}_{i}\right\|=1$.

Although Eq. (8) bounds the operator norm that is of interest, the rhs depends on this unknown operator norm. Nevertheless, one can use the generalized Grönwall's inequality [63] to show that $\left\|\hat{\gamma}_{i}^{B}(t)\right\| \leq \bar{\gamma}_{i}^{B}(t)$, where $\bar{\gamma}_{i}^{B}(t)$ is the solution to Eq. (8) with the inequality replaced by equality:

$$
\bar{\gamma}_{i}^{B}(t)=\bar{\gamma}_{i}^{B}(0)+2 \sum_{j:\langle i j\rangle \in G}\left|h_{j}\right| \int_{0}^{t} \bar{\gamma}_{j}^{B}\left(t^{\prime}\right) d t^{\prime},
$$

where $\bar{\gamma}_{i}^{B}(0)=\left\|\hat{\gamma}_{i}^{B}(0)\right\|$. Differentiating Eq. (9), one obtains the first-order linear differential equation

$$
\dot{\bar{\gamma}}_{i}^{B}(t)=2 \sum_{j:\langle i j\rangle \in G}\left|h_{j}\right| \bar{\gamma}_{j}^{B}(t)
$$

with initial condition

$$
\bar{\gamma}_{i}^{B}(0)=\left\|\left[\hat{\gamma}_{i}, \hat{B}\right]\right\|
$$

We define the support of operator $\hat{B}$ on the commutativity graph as $S(\hat{B})=\left\{i \mid\left[\hat{\gamma}_{i}, \hat{B}\right] \neq 0\right\}$. Then the initial condition can be simplified as $\bar{\gamma}_{i}^{B}(0)=2\|\hat{B}\|(i \in S(B))$, where the notation $(P)$ is defined as $(P)=1$ if $P$ is true and $(P)=0$ if $P$ is false, for an arbitrary statement $P$.

The differential equation Eq. (10) gives an upper bound for the unequal time commutator of a Hamiltonian term with an arbitrary local operator. To obtain the upper bound of $\hat{A}^{B}(t)=[\hat{A}(t), \hat{B}(0)]$, where $\hat{A}$ is an operator not in the Hamiltonian, we can perform similar calculations in Eqs. (5)-(8) to get

$$
\left\|\hat{A}^{B}(t)\right\|-\left\|\hat{A}^{B}(0)\right\| \leq \int_{0}^{t} \sum_{i \in S(\hat{A})} 2\|\hat{A}\|\left|h_{i}\right| \bar{\gamma}_{i}^{B}\left(t^{\prime}\right) d t^{\prime} .
$$

For later convenience, we introduce Green's function as a useful tool to discuss solutions to Eq. (10). First notice that Eq. (10) can be rewritten in a symmetric form

$$
\dot{\bar{\Gamma}}_{i}^{B}(t)=2 \sum_{j:\langle i j\rangle \in G} \sqrt{\left|h_{i}\right|\left|h_{j}\right|} \bar{\Gamma}_{j}^{B}(t)
$$

where $\bar{\Gamma}_{i}^{B}(t)=\sqrt{h_{i}} \bar{\gamma}_{i}^{B}(t)$. We denote the real symmetric coefficient matrix as $H_{i j}=2 \sqrt{\left|h_{i}\right|\left|h_{j}\right|}(\langle i j\rangle \in G)$. Green's function $G_{i j}(t)$ is defined as the solution to the differential equation

$$
\dot{G}_{i j}(t)=\sum_{k:\langle i k\rangle \in G} H_{i k} G_{k j}(t)
$$

with initial condition $G_{i j}(0)=\delta_{i j}$, so any initial value problem can be obtained by linear combinations of Green's functions:

$$
\bar{\Gamma}_{i}^{B}(t)=\sum_{j} G_{i j}(t) \bar{\Gamma}_{j}^{B}(0)
$$

and, therefore, the LR bound of $\left\|\left[\hat{\gamma}_{i}(t), \hat{B}_{Y}(0)\right]\right\|$ can be expressed as

$$
\left\|\left[\hat{\gamma}_{i}(t), \hat{B}_{Y}(0)\right]\right\| \leq \sum_{j \in Y} G_{i j}(t) \sqrt{\frac{h_{j}}{h_{i}}} 2\|\hat{B}\|,
$$

where $Y=S(\hat{B})$ is the support of operator $\hat{B}$ on the commutativity graph (from now on we use $\hat{A}_{X}$ to mean that operator $\hat{A}$ has support $X$ on the commutativity graph). The LR bound for the unequal time commutator between arbitrary local operators $\left\|\left[\hat{A}_{X}(t), \hat{B}_{Y}(0)\right]\right\|$ can be obtained by inserting Eq. (16) into Eq. (12):

$$
\begin{aligned}
& \left\|\left[\hat{A}_{X}(t), \hat{B}_{Y}(0)\right]\right\|-\left\|\left[\hat{A}_{X}(0), \hat{B}_{Y}(0)\right]\right\| \\
& \quad \leq 4\|\hat{A}\|\|\hat{B}\| \int_{0}^{t} \sum_{i \in X, j \in Y} \sqrt{\left|h_{i} h_{j}\right|} G_{i j}\left(t^{\prime}\right) d t^{\prime} .
\end{aligned}
$$

Equations (14) and (17) [or Eqs. (10) and (12)] are the main results of this section, which upper bound the unequal time commutators by the solution to a set of linear differential equations. Note that the bound Eq. (17) can in general be efficiently computed. One can obtain $G_{i j}(t)$ by solving Eq. (14), a set of $N_{H}$ linear coupled ordinary differential equations, where $N_{H}$ is the number of terms in the Hamiltonian, which grows linearly with system size in locally interacting systems. This is a substantial reduction from solving the original many-body problem, whose cost in general grows exponentially with the system size. Furthermore, in translation invariant systems, we can solve these equations by a Fourier transform, which allows us to derive further simplified analytic upper bounds, as shown in the next section. 
We end this section by commenting on the issue noted at the end of Sec. II A. As we mention there, we can write the same Hamiltonian in the form of Eq. (4) in different ways, leading to different commutativity graphs. The differential equations and the resulting LR bound are different as well. One may ask how to choose a decomposition of the Hamiltonian $\stackrel{H}{H}$ so that the resulting LR bound is tightest. While there is not a general statement about which decomposition is best, for spin-1/2 and fermionic systems we know a particularly good choice of decomposition that in many cases give us the tightest bound. For such systems, it is always possible to write the Hamiltonian in the form of Eq. (4) $[64,65]$ if we require that $\hat{\gamma}_{i}^{2}=1$ and $\hat{\gamma}_{i}, \hat{\gamma}_{j}$ for $i \neq j$ either commute or anticommute. More precisely, the algebraic relations of $\left\{\hat{\gamma}_{i}\right\}$ are related to their commutativity graph $G$ as follows:

$$
\begin{aligned}
\hat{\gamma}_{i}^{2}=1, & \text { for } \forall i \in G, \\
\left\{\hat{\gamma}_{i}, \hat{\gamma}_{j}\right\}=0, & \text { for }\langle i j\rangle \in G, \\
{\left[\hat{\gamma}_{i}, \hat{\gamma}_{j}\right]=0, } & \text { for }\langle i j\rangle \notin G .
\end{aligned}
$$

For example, in spin- $1 / 2$ systems $\hat{\gamma}_{i}$ can be chosen as products of Pauli matrices, while in fermionic systems they are products of Majorana fermion operators. More specific examples are given in Sec. V. If the additional constraints Eq. (18) still fail to specify the decomposition uniquely, we choose [among those satisfying Eq. (18)] a decomposition such that the total number of $\hat{\gamma}_{i}$ terms is minimal. We call it a minimal Clifford decomposition. The LR bound derived from this decomposition is typically tightest since the triangle inequality $\|[A, B]\| \leq 2\|A\|\|B\|$ (which is used many times in our derivation) is saturated when $\{A, B\}=0$. One can also show that the resulting LR bound has the tightest small- $t$ exponent in typical models, as discussed in Sec. IV.

\section{Alternative treatment for interacting fermions}

Our previous analysis applies to the fermionic case as well, since our derivations did not rely on any special property of the Hamiltonians beyond being locally interacting. However, for the case of interacting fermions in an arbitrary $d$-dimensional lattice, we can use a slightly different method, which sometimes gives us a tighter LR bound.

In the following we consider an arbitrary locally interacting fermionic system with up to quartic terms, but the method generalizes straightforwardly to systems with higher-order interacting terms. We use the Majorana representation for convenience, where the Hamiltonian can in general be written as

$$
\hat{H}=\sum_{i<j} t_{i j} i \hat{c}_{i} \hat{c}_{j}+\sum_{i<j<k<l} U_{i j k l} \hat{c}_{i} \hat{c}_{j} \hat{c}_{k} \hat{c}_{l}
$$

where $t_{i j}$ and $U_{i j k l}$ are totally antisymmetric, and the Majorana operators satisfy

$$
\begin{aligned}
\hat{c}_{i}^{\dagger} & =\hat{c}_{i}, \\
\left\{\hat{c}_{i}, \hat{c}_{j}\right\} & =2 \delta_{i j} .
\end{aligned}
$$

We first upper bound the norm of the unequal time anticommutator $\hat{c}_{i j}(t)=\left\{\hat{c}_{i}(t), \hat{c}_{j}(0)\right\}$. The Heisenberg equation for the operator $\hat{c}_{i}(t)=e^{i \hat{H} t} \hat{c}_{i} e^{-i \hat{H} t}$ is

$$
i \dot{\hat{c}}_{i}(t)=2 \sum_{j} t_{i j} \hat{c}_{j}(t)+2 \sum_{j<k<l} U_{i j k l} \hat{c}_{j}(t) \hat{c}_{k}(t) \hat{c}_{l}(t) .
$$

Using Eq. (21), we have the evolution equation for $\hat{c}_{i m}(t)$

$$
\begin{aligned}
i \dot{\hat{c}}_{i m}(t)= & 2 \sum_{j<k<l} U_{i j k l}\left\{\hat{c}_{j}(t) \hat{c}_{k}(t) \hat{c}_{l}(t), \hat{c}_{m}(0)\right\} \\
& +2 \sum_{j} t_{i j} i \hat{c}_{j m}(t) .
\end{aligned}
$$

The first term of Eq. (22) can be expanded into the sum of three terms using $\{a b c, d\}=a b\{c, d\}+a\{b, d\} c+$ $\{a, d\} b c$. Therefore,

$$
\begin{aligned}
\left\|\hat{c}_{i m}(t)\right\|-\left\|\hat{c}_{i m}(0)\right\| \leq & \int_{0}^{t}\left\|\dot{\hat{c}}_{i m}\left(t^{\prime}\right)\right\| d t^{\prime} \\
\leq & 2 \int_{0}^{t} d t^{\prime} \sum_{j<k<l}\left|U_{i j k l}\right| \\
& \times\left\|\hat{c}_{j m}\left(t^{\prime}\right)+\hat{c}_{k m}\left(t^{\prime}\right)+\hat{c}_{l m}\left(t^{\prime}\right)\right\| \\
& +2 \int_{0}^{t} \sum_{j}\left|t_{i j}\right|\left\|\hat{c}_{j m}\left(t^{\prime}\right)\right\| d t^{\prime} .
\end{aligned}
$$

Equation (23) is the analog of Eq. (8). Therefore, using the same argument as we use in Sec. II B, we can conclude that $\left\|\hat{c}_{i m}(t)\right\| \leq c_{i m}(t)$ where $c_{i m}(t)$ is the solution to the differential equation

$$
\begin{aligned}
\dot{c}_{i m}(t)= & 2 \sum_{j<k<l}\left|U_{i j k l}\right|\left[c_{j m}(t)+c_{k m}(t)+c_{l m}(t)\right] \\
& +2 \sum_{j}\left|t_{i j}\right| c_{j m}(t)
\end{aligned}
$$

with initial condition $c_{i m}(0)=\left\|\hat{c}_{i m}(0)\right\|=2 \delta_{i m}$. Once we know the LR bound for $\left\{\hat{c}_{i}(t), \hat{c}_{j}(0)\right\}$, we can calculate LR bounds for arbitrary local operators using identities like $[a b, c d]=a\{b, c\} d-a c\{b, d\}+\{a, c\} d b-c\{a, d\} b$, since an arbitrary local operator can be expressed as products of basic Majorana operators $c_{j}$.

Equation (24) takes a similar form as Eq. (10) and can be treated similarly. For example, Green's function method 
in Sec. II B can also be applied, and the analyses in the following two sections apply to both equations equally well. We currently do not have a general criteria to determine which equation leads to a tighter LR bound for a specific fermionic system, and the results have to be compared case by case. But compared to LR bounds obtained prior to the current paper, both methods give improved LR speeds, correct power-law growth of correlations at short time, and the superexponentially decaying tail. See the application to the FH model in Sec. V D for a detailed comparison.

\section{ANALYTIC BOUNDS IN TRANSLATION INVARIANT SYSTEMS}

In the last section we upper bound the unequal time commutator by the solution to an integral inequality [Eq. (17)] with the integrand given by the solution to a set of linear differential equations [Eq. (14)]. In translation invariant systems, we can obtain the solution to these equations in the form of a Fourier integral, and derive a simpler, fully analytic upper bound by methods of complex analysis.

We start our discussion by considering Eq. (14) in a general periodic lattice structure and obtain a formal solution in terms of a Fourier integral. Suppose there are $l$ vertices in a primitive unit cell, labeled by index $\alpha=$ $1, \ldots, l$. We use capital $I$ to label unit cells with coordinate labeled by $\vec{r}_{I}$ [for example, if $\left\{\boldsymbol{\alpha}_{1}, \ldots, \boldsymbol{\alpha}_{d}\right\}$ is a set of primitive lattice translation vectors, then coordinate $\vec{r}_{I}=$ $\left(n_{1}, n_{2}, \ldots, n_{d}\right), n_{a} \in \mathbb{Z}, a=1, \ldots, d$ labels the primitive cell at physical position $\mathbf{R}_{I}=\sum_{a=1}^{d} n_{a} \boldsymbol{\alpha}_{a}$ ], so that every vertex $i$ can be labeled by a pair $(I, \alpha)$. Taking advantage of translation invariance, we can use the Fourier integral representation of Green's function $G_{i j}(t) \equiv G_{I \alpha ; J \beta}(t)$,

$$
G_{i j}(t) \equiv \int_{-\pi}^{\pi} \frac{d^{d} k}{(2 \pi)^{d}} G_{\alpha \beta}^{(\vec{k})}(t) e^{i \vec{k} \cdot\left(\vec{r}_{I}-\vec{r}_{J}\right)} .
$$

[Notice that other discrete symmetries of the lattice (such as inversion, reflection, discrete rotation, etc.) do not play a significant role in this context, so we are using integer coordinates of unit cells and consequently the Fourier momentum simply lies in $(-\pi, \pi]^{d}$ rather than the first Brillouin zone in typical solid-state contexts.] Inserting Eq. (25) into Eq. (14) we get

$$
\dot{G}_{\alpha \beta}^{(\vec{k})}(t)=\sum_{\gamma=1}^{l} H_{\alpha \gamma}^{(\vec{k})} G_{\gamma \beta}^{(\vec{k})}(t),
$$

where the $l \times l$ coefficient matrix $H_{\alpha \beta}^{(\vec{k})}$ is the Fourier transform of $H_{I \alpha ; J \beta}$ (notice that due to translation invariance $H_{I \alpha ; J \beta}$ only depends on $\left.\vec{r}_{I}-\vec{r}_{J}\right)$ :

$$
H_{\alpha \beta}^{(\vec{k})}=\sum_{J} H_{I \alpha ; J \beta} e^{-i \vec{k} \cdot\left(\vec{r}_{I}-\vec{r}_{J}\right)} .
$$

The formal solution to Eq. (26) is $G_{\alpha \beta}^{(\vec{k})}(t)=\left[e^{H^{(\vec{k})} t}\right]_{\alpha \beta}$, where $H^{(\vec{k})}$ is considered as an $l \times l$ matrix.

We now derive a simple analytic upper bound for the Fourier integral of Eq. (25) using methods of complex analysis. Since the integrand of Eq. (25) is a complex analytic function of $\vec{k}$ except at infinity, and since the integrand is periodic in shifts of the real axis by $2 \pi$, we can send $\vec{k} \rightarrow \vec{k}+i \vec{\kappa}$ without changing the result of the integral, as illustrated in Fig. 2. Therefore, we have

$$
\begin{aligned}
\left|G_{i j}(t)\right| & =\left|\int_{-\pi}^{\pi} \frac{d^{d} k}{(2 \pi)^{d}}\left[e^{H^{(\vec{k}+i \vec{\kappa})} t}\right]_{\alpha \beta} e^{i \vec{k} \cdot \vec{r}-\vec{\kappa} \cdot \vec{r}}\right| \\
& \leq \int_{-\pi}^{\pi} \frac{d^{d} k}{(2 \pi)^{d}}\left|\left[e^{H^{(\vec{k}+i \vec{\kappa})} t}\right]_{\alpha \beta}\right| e^{-\vec{\kappa} \cdot \vec{r}} \\
& \leq\left|\left[e^{H^{(i \vec{k})} t}\right]_{\alpha \beta}\right| e^{-\vec{\kappa} \cdot \vec{r}} \\
& \leq c_{\vec{\kappa}} e^{\omega_{m}(i \vec{k}) t-\vec{\kappa} \cdot \vec{r}},
\end{aligned}
$$

where $\vec{r}=\vec{r}_{I}-\vec{r}_{J}$, in the second line we use the triangle inequality for the integral, and in the third line we use the inequality $\left|\left[e^{H^{(\vec{k}+\vec{k})} t}\right]_{\alpha \beta}\right| \leq\left|\left[e^{H^{(i \vec{k})} t}\right]_{\alpha \beta}\right|$ [which follows from the triangle inequality since the coefficients $H_{I \alpha ; J \beta}$ in Eq. (27) are nonnegative]. In the last line we use the inequality $\left|\left[e^{H^{(i \vec{k})} t}\right]_{\alpha \beta}\right| \leq\left\|e^{H^{(\vec{k})} t}\right\| \leq c_{\vec{\kappa}} e^{\omega_{m}(\vec{k}) t}$, where $\omega_{m}(i \vec{k})$ is the eigenvalue of $H^{(i \vec{k})}$ with largest magnitude $\left[\omega_{m}(i \vec{\kappa})\right.$ must be real positive according to the PerronFrobenius theorem], $c_{\vec{\kappa}}$ is a function of $\vec{\kappa}$ independent of $t$, and the last inequality can be proved by diagonalizing $H^{(i \vec{k})}$ into Jordan canonical form. Therefore, we arrive at the following important result:

$$
\left|G_{i j}(t)\right| \leq c_{\vec{\kappa}} e^{\omega_{m}(i \vec{\kappa}) t-\vec{\kappa} \cdot \vec{r}}, \quad \forall \vec{\kappa} \in \mathbb{R}^{d} .
$$

While this expression looks quite similar to previous LR bounds with exponential decaying tail, described by Eq. (1), the point here is that we can choose $\vec{\kappa}=\vec{\kappa}(\vec{r}, t)$ to depend on $\vec{r}, t$ to minimize the rhs at each point in space time. This makes the bound decaying faster than exponential at large distance, as we prove in Sec. IV, and see in examples in Sec. V.

We end this subsection by giving an explicit formula for the LR speed that emerges from this bound. The LR speed of Green's function $G_{i j}(t)$ (considered as a function of $\vec{r}, t)$ in Eq. (25) is, by definition, the speed at which $r$ must change as a function of $t$ in order for $G_{i j}(t)$ 's magnitude to stay constant. While Eq. (25) is not simple enough to exactly extract the LR speed, we can get an upper bound for the LR speed from the upper bound of $G_{i j}(t)$ in Eq. (29). If we choose $\vec{\kappa}=\vec{\kappa}_{0} \equiv \operatorname{sgn}(\vec{r}) \kappa_{0}$, where $\operatorname{sgn}(\vec{r}) \equiv\left(\operatorname{sgn}\left(r_{1}\right), \operatorname{sgn}\left(r_{2}\right), \ldots, \operatorname{sgn}\left(r_{d}\right)\right)$, we get the 


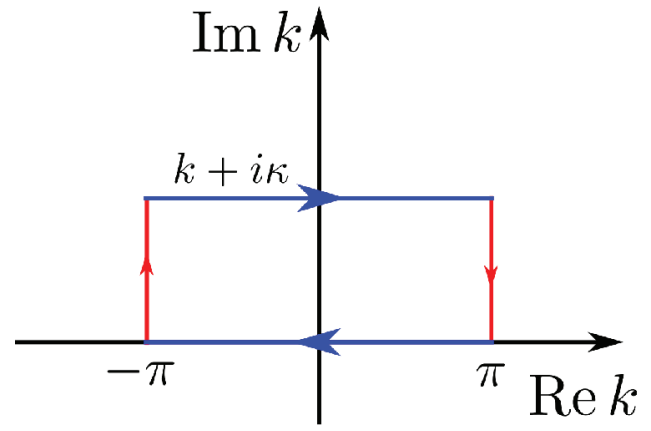

FIG. 2. Since the integrand of Eq. (25) is a complex analytic function of $\vec{k}$ except at infinity, the integration along the above contour is zero. Since the integrand is a periodic function of $\vec{k}$ with period $2 \pi$, the integral on two vertical arrows (colored red) cancel. The net result is that in Eq. (25) we can send $\vec{k} \rightarrow \vec{k}+i \vec{\kappa}$ without changing the result of the integral.

old form of LR bound with exponential decaying tail

$$
|G(\vec{r}, t)| \leq c_{\vec{\kappa}_{0}} e^{\omega_{m}\left(\vec{i}_{0}\right) t-\kappa_{0}|\vec{r}|},
$$

where $|\vec{r}|=\sum_{j=1}^{d} r_{j}$ is the Manhattan distance between the corresponding unit cells. Therefore, the LR speed of $|G(\vec{r}, t)|$ must be upper bounded by an optimal choice of $\kappa_{0}$ :

$$
v_{\mathrm{LR}} \leq \min _{\kappa_{0}>0} \frac{\omega_{m}\left(i \vec{\kappa}_{0}\right)}{\kappa_{0}} .
$$

Notice that while $\vec{\kappa}_{0}$ depends on $\operatorname{sgn}(\vec{r})$, in typical models $\omega_{m}\left(i \vec{\kappa}_{0}\right)$ does not depend on $\operatorname{sgn}(\vec{r})$ [see Sec. V]. In the case that $\omega_{m}\left(i \vec{\kappa}_{0}\right)$ does depend on $\operatorname{sgn}(\vec{r})$, Eq. (31) represents a directional LR speed, and to get the overall LR speed we should take the maximum over all possible $2^{d}$ values of $\operatorname{sgn}(\vec{r})$.

It is immediately clear from Eq. (17) that the LR speed of Green's function gives an upper bound for the LR speed of the unequal time commutator between any local operators $\left\|\left[\hat{A}_{X}(t), \hat{B}_{Y}(0)\right]\right\|$, since, by inserting Eq. (30) into Eq. (17), we get

$$
\left\|\left[\hat{A}_{X}(t), \hat{B}_{Y}(0)\right]\right\| \leq C e^{\omega_{m}\left(i \vec{\kappa}_{0}\right) t-\kappa_{0} r_{X Y}},
$$

where $C=4 c_{\vec{\kappa}_{0}}\|\hat{A}\|\|\hat{B}\| \sqrt{h_{X} h_{Y}} / \omega_{m}\left(i \vec{\kappa}_{0}\right), h_{X} \equiv \max _{i \in X} h_{i}$, $r_{X Y}=\min _{i \in X, j \in Y} r_{i j}$ and we assume $X \cap Y=\emptyset$. This proves that $\left\|\left[\hat{A}_{X}(t), \hat{B}_{Y}(0)\right]\right\|$ has the same LR speed $v_{\mathrm{LR}}$.

Equation (31) is one of the main results of our paper, for upper bounding the LR speed of a locally interacting many-body Hamiltonian. Compared to previous methods for upper bounding $v_{\mathrm{LR}}$, for example the methods given in Ref. [2] or Ref. [3], our formula Eq. (31) is not only simpler but also gives much tighter bounds, as shown in specific examples in Sec. V.

\section{SIMPLE LR BOUND FOR AN ARBITRARY GRAPH}

In this section we prove the simple LR bound in Eq. (3) for an arbitrary locally interacting system. The only assumption we make about the system is that the commutativity graph $G$ has an upper bound on the degree of vertices and the weight $H_{i j}$ of the edges. We first express Green's function in an arbitrary graph as a Taylor series of $t$, illustrate the graph-theoretical meaning of the expansion coefficients, and then use this expansion to prove the LR bound.

The formal solution to Eq. (14) is

$$
G_{i j}(t)=\left[e^{H t}\right]_{i j}=\sum_{n \geq 0} G_{i j}^{(n)} \frac{t^{n}}{n !},
$$

where $G_{i j}^{(n)}=\left[H^{n}\right]_{i j}$. Notice that $H_{i j}$ can be considered as the adjacency matrix of the weighted commutativity graph $G$ with the weight of the edge $(i, j)$ being $H_{i j}$. In this way, the meaning of $G_{i j}^{(n)}$ is the sum of the weights of all possible paths in $G$ connecting vertices $i$ and $j$, where the weight of a path is the product of the weights of all its edges. (Note: there can be duplicate edges in a path, and the weight of path $p$ is $w_{p}=\prod_{e \in p} w_{e}^{d_{e}}$ where $d_{e}$ is the multiplicity of edge $e$.) Therefore, we have $G_{i j}^{(n)}=0$ for $n<d_{i j}$ where $d_{i j}$ is the graph theoretical distance (length of the shortest path) between $i$ and $j$. In summary we have

$$
G_{i j}(t)=\sum_{n \geq d_{i j}} G_{i j}^{(n)} \frac{t^{n}}{n !} .
$$

We now prove the LR bound in Eq. (3). First let us prove that there exists a velocity $u$ such that for all pairs of vertices $i, j$ we have

$$
G_{i j}(t) \leq c\left(u t / d_{i j}\right)^{d_{i j}}, \quad \text { for } t \leq d_{i j} / u,
$$

for some positive constant $c$. Inserting Eq. (34), the above inequality is equivalent to

$$
\sum_{n \geq d_{i j}} G_{i j}^{(n)} \frac{t^{n-d_{i j}}}{n !} \leq c\left(u / d_{i j}\right)^{d_{i j}}, \quad \text { for } t \leq d_{i j} / u .
$$

Since the $H_{i j}$ are nonnegative, the coefficients $G_{i j}^{(n)}$ are nonnegative, so the lhs of Eq. (36) is a nondecreasing function of $t$ while the rhs is independent of $t$. Therefore, we only need to prove that the inequality holds for $t=d_{i j} / u$ :

$$
\sum_{n \geq d_{i j}} G_{i j}^{(n)} \frac{\left(d_{i j} / u\right)^{n-d_{i j}}}{n !} \leq c\left(u / d_{i j}\right)^{d_{i j}},
$$


which can be simplified to

$$
G_{i j}\left(d_{i j} / u\right) \leq c .
$$

This is exactly the statement that Green's function has a finite LR velocity $u$.

One can show that Eq. (38) holds extremely generally, requiring only locality and bounded magnitude of the $H_{i j}$. For translation invariant systems, we already prove this in the last section [see Eq. (30)], except that distance is measured differently in Eqs. (30) and (38): in the former case we use the Manhattan distance between unit cells while in the latter case we use graph-theoretical distance in the commutativity graph, so $u$ is in general different from $v_{\mathrm{LR}}$. But since $c_{1} \leq d_{i j} / r_{i j} \leq c_{2}$ for some constants $c_{1}, c_{2}$ (this follows straightforwardly from the fact that each real-space unit cell is associated with a finite number of terms in $\hat{H}$ ), existence of a finite $v_{\mathrm{LR}}$ guarantees existence of $u$. In the next section we show in some specific models how to find a tight $u$. For the general case without translation invariance, existence of a finite $u$ can be proved under the assumption that the weighted graph $G$ has an upper bound on the degree of vertices and the weights of the edges, that is, every vertex has at most $\lambda$ edges and the weights satisfy $H_{i j} \leq h$ for all pairs of neighboring vertices $i, j$. With these assumptions we have $G_{i j}^{(n)} \leq h^{n} \lambda^{n}, \forall i, j \in G, n \geq 0$. Inserting into Eq. (34), we have

$$
\begin{aligned}
G_{i j}(t) & \leq \sum_{n \geq d_{i j}} \frac{(\lambda h t)^{n}}{n !} \\
& \leq \sum_{n \geq d_{i j}} \frac{(\lambda h t)^{n}}{e(n / e)^{n}} \\
& \leq \sum_{n \geq d_{i j}}\left(\lambda h t e / d_{i j}\right)^{n} / e \\
& =\left(\lambda h t e / d_{i j}\right)^{d_{i j}} \frac{1 / e}{1-\lambda h t e / d_{i j}},
\end{aligned}
$$

for $t<d_{i j} / \lambda h e$. This proves Eq. (38) if we choose, for example, $u=2 \lambda$ he.

Now we use the bound Eq. (35) on $G_{i j}(t)$ to bound a general unequal-time commutator $\left\|\left[\hat{A}_{X}(t), \hat{B}_{Y}(0)\right]\right\|$. Inserting Eq. (35) into Eq. (17), we have

$$
\begin{aligned}
& \left\|\left[\hat{A}_{X}(t), \hat{B}_{Y}(0)\right]\right\|-\left\|\left[\hat{A}_{X}(0), \hat{B}_{Y}(0)\right]\right\| \\
& \leq 4 c_{\vec{\kappa}_{0}}\|\hat{A}\|\|\hat{B}\| \int_{0}^{t} \sum_{i \in X, j \in Y} \sqrt{h_{i} h_{j}}\left(\frac{u t^{\prime}}{d_{i j}}\right)^{d_{i j}} d t^{\prime}
\end{aligned}
$$

$$
\begin{aligned}
& \leq 4 c_{\vec{\kappa}_{0}}\|\hat{A}\|\|\hat{B}\| \frac{e}{u} \sum_{i \in X, j \in Y} \sqrt{h_{i} h_{j}}\left(\frac{u t}{d_{i j}+1}\right)^{d_{i j}+1} \\
& \leq 4 c_{\kappa_{0}}\|\hat{A}\|\|\hat{B}\| \frac{e}{u} h_{X Y}\left(\frac{u t}{d_{X Y}+1}\right)^{d_{X Y}+1}
\end{aligned}
$$

for $t \leq d_{X Y} / u$, where $h_{X Y}$ is a geometric factor defined as

$$
h_{X Y}=\sum_{i \in X, j \in Y} \sqrt{h_{i} h_{j}} e^{d_{X Y}-d_{i j}} .
$$

In the third line of Eq. (40) we use the inequality $(1+$ $\left.1 / d_{i j}\right)^{d_{i j}} \leq e$, and in the last line we use $(u t / x)^{x} \leq$ $(u t / y)^{y} e^{y-x}$ for $t \leq y / u \leq x / u$ [this inequality holds since the function $(u t / x)^{x} e^{x}$ is decreasing in $x$ for $\left.x \geq u t\right]$. For $t>d_{X Y} / u$ we have the trivial bound $\left\|\left[\hat{A}_{X}(t), \hat{B}_{Y}(0)\right]\right\| \leq$ $2\|\hat{A}\|\|\hat{B}\| \leq 2 e\|\hat{A}\|\|\hat{B}\|\left[u t /\left(d_{X Y}+1\right)\right]^{d_{X Y}+1}$. In summary we have

$$
\left\|\left[\hat{A}_{X}(t), \hat{B}_{Y}(0)\right]\right\| \leq C\left(\frac{u t}{d_{X Y}+1}\right)^{d_{X Y}+1},
$$

where $C=2 e \max \left\{1,2 c_{\vec{\kappa}_{0}}\left(h_{X Y} / u\right)\right\}\|A\|\|B\|$ for $X \cap Y=\emptyset$ and $\forall t \geq 0$. This completes the proof of the LR bound in Eq. (3). [Eq. (42) is actually a slightly tighter version].

The LR bound in Eq. (42) substantially and qualitatively improves previous LR bounds, not only because it has the superexponential decaying tail $e^{-r \ln r}$, but also because it has much tighter small- $t$ exponent, and a much tighter LR speed, as seen in examples in the next section. The small- $t$ exponent $\eta(\vec{r})$ of a bound $f(\vec{r}, t)$ is defined by its limiting behavior $f(\vec{r}, t) \propto t^{\eta(\vec{r})}$ as $t \rightarrow 0$. We now argue that if a minimal Clifford decomposition [as defined below Eq. (18)] of the Hamiltonian is used in Eq. (4), then the bound in Eq. (42) has a small-t exponent that generically agree with the exact result, and thus are the tightest possible. For simplicity, consider the LR bound for $\left[\hat{\gamma}_{i}(t), \hat{\gamma}_{j}(0)\right]$. The Baker-Campbell-Hausdorff formula gives

$$
\begin{aligned}
{\left[\hat{\gamma}_{i}(t), \hat{\gamma}_{j}\right] } & =\left[\sum_{n \geq 0} \frac{(i t)^{n}}{n !} \operatorname{ad}_{\hat{H}}^{n}\left(\hat{\gamma}_{i}\right), \hat{\gamma}_{j}\right] \\
& =\frac{(2 i t)^{d_{i j}-1}}{\left(d_{i j}-1\right) !} \sum_{p \in P_{i j}} \hat{\gamma}_{i} \hat{S}_{p} \hat{\gamma}_{j}+O\left(t^{d_{i j}}\right),
\end{aligned}
$$

where $\operatorname{ad}_{\hat{H}}$ is the adjoint map of $\hat{H}$ acting on the space of operators defined as $\operatorname{ad}_{\hat{H}}(\hat{B}) \equiv[\hat{H}, \hat{B}], P_{i j}$ is the set of shortest paths connecting $i$ and $j$ (note: by our convention a path $p \in P_{i j}$ does not contain $i$ and $j$ ), the string operator $\hat{S}_{p}$ along a path $p \in P_{i j}$ is defined as $\hat{S}_{p}=\prod_{k \in p} h_{k} \hat{\gamma}_{k}$ (with a suitable ordering). Therefore, the small- $t$ exponent is $\eta_{i j}=d_{i j}-1$ provided that the sum on the second line of Eq. (43) is nonzero. A sufficient condition for this to be 
true is if no two string operators $\hat{S}_{p}$ and $\hat{S}_{p^{\prime}}$ along different paths $p \neq p^{\prime}$ are proportional to each other [notice that each individual term $\hat{\gamma}_{i} \hat{S}_{p} \hat{\gamma}_{j}$ is a product of invertible operators (due to the relation $\hat{\gamma}_{j}^{2}=1$ ) and is, therefore, always nonzero]. This condition is satisfied by typical models such as the TFIM, FH model, the Heisenberg XYZ model, and we believe that it is automatically satisfied by the minimal Clifford decomposition of the Hamiltonian. Therefore, the small- $t$ exponent $d_{X Y}+1$ of the bound in Eq. (42) is saturated by exact results, since when $X=S\left(\hat{\gamma}_{i}\right), Y=S\left(\hat{\gamma}_{j}\right)$, we have $d_{i j}-1=d_{X Y}+1$. This has never been achieved in previous LR bounds, where the small- $t$ exponent is typically just $\eta(\vec{r})=1$, as in Eq. (1) for example [66].

\section{EXAMPLES}

In this section, we apply the techniques developed above to calculate the LR velocity for the bound of Eq. (3) explicitly for five models as examples: the $d$-dimensional TFIM (Sec. V A), the spin- $S$ Heisenberg model (Sec. V B), the $N$-state truncated BH model (Sec. VC), the $\mathrm{SU}(N) \mathrm{FH}$ model (Sec. V D), and Wen's quantum rotor model (Sec. $\mathrm{VE}$ ). The techniques are general and can be straightforwardly applied to arbitrary models. All these examples demonstrate significant quantitative improvements over the previous LR speeds, and in a number of limits (e.g., large- $d$, large- $S$, or large- $N$ ) our bounds are qualitatively tighter for having better scalings. We introduce techniques to further tighten the LR velocity in Sec. VI.

\section{A. The $d$-dimensional TFIM}

In the following we first derive a bound in the familiar spin- $1 / 2$ case, and then extend this result to arbitrary spin in Sec. V A 2.

\section{Spin-1/2 case}

The Hamiltonian for the $d$-dimensional hypercubic lattice Ising model with a transverse field is

$$
\hat{H}=-J \sum_{\vec{r}, 1 \leq j \leq d} \hat{\gamma}_{\vec{r}, j}-h \sum_{\vec{r}} \hat{\gamma}_{\vec{r}, 0}
$$

with $\hat{\gamma}_{r, j}=\hat{\sigma}_{\vec{r}}^{z} \hat{\sigma}_{\vec{r}+\hat{e}_{j}}^{z}$ where $\hat{e}_{j}$ is the unit vector in the $j$ th direction, and $\hat{\gamma}_{r}, 0=\hat{\sigma}_{\vec{r}}^{x}$. We assume $J \geq 0, h \geq 0$ (the resulting LR bound only depends on $|J|$ and $|h|)$. We write the Hamiltonian in this form so that Eq. (18) is satisfied. It is easily verified that the square of each term is equal to unity $\hat{\gamma}_{\vec{r}, \alpha}^{2}=1, \alpha=0,1,2, \ldots, d$, and any two terms either commute or anticommute. Figure 3 shows the commutativity graph of this model for the $d=2$ case.

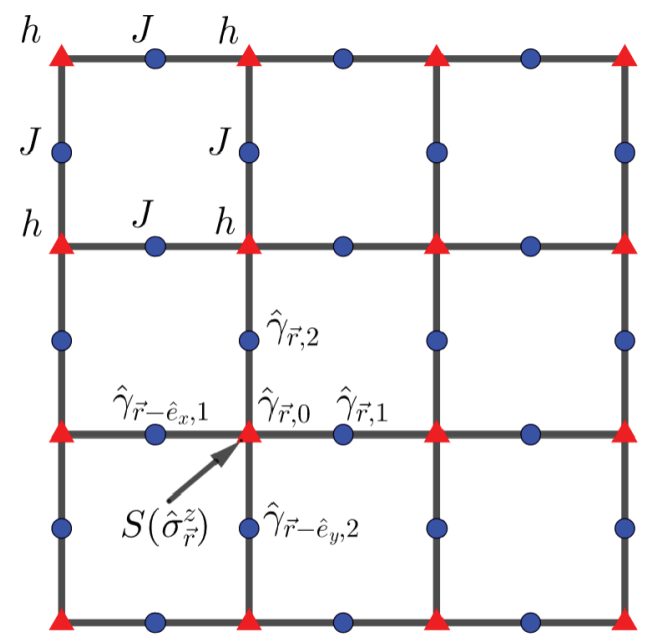

FIG. 3. Commutativity graph $G$ of the 2D TFIM. Each term (a local product of Pauli matrices) of the Hamiltonian is represented by a vertex. Any pair of terms either commutes or anticommutes. We link two vertices by an edge if the corresponding operators anticommute. The coefficient of each term is drawn adjacent to the corresponding vertices. The arrow points to the region $S\left(\hat{\sigma}_{\vec{r}}^{z}\right)$, the support of operator $\hat{\sigma}_{\vec{r}}^{z}$, which consists of only one point in this case.

We can use the general Fourier integral representation of Green's function in Eq. (25)

$$
G_{\alpha \beta}(\vec{r}, t) \equiv \int_{-\pi}^{\pi} \frac{d^{d} k}{(2 \pi)^{d}}\left\{\exp \left[H^{(\vec{k})} t\right]\right\}_{\alpha \beta} e^{i \vec{k} \cdot\left(\vec{r}_{I}-\vec{r}_{J}\right)}
$$

where $H^{(\vec{k})}$, defined in Eq. (27), is in this case a $(d+1) \times$ $(d+1)$ matrix

$$
H^{(\vec{k})}=2 \sqrt{J h}\left[\begin{array}{cccc}
0 & 1+e^{-i k_{1}} & \ldots & 1+e^{-i k_{d}} \\
1+e^{i k_{1}} & 0 & 0 & 0 \\
\vdots & 0 & 0 & 0 \\
1+e^{i k_{d}} & 0 & 0 & 0
\end{array}\right],
$$

so that

$$
\begin{aligned}
\exp \left[H^{(\vec{k})} t\right]= & \frac{\sinh [\omega(\vec{k}) t]}{\omega(\vec{k})} H^{(\vec{k})}+\cosh [\omega(\vec{k}) t] \mathbb{1} \\
& +P_{0}\{1-\cosh [\omega(\vec{k}) t]\}
\end{aligned}
$$

where $\mathbb{1}$ is the identity matrix and

$$
\begin{aligned}
P_{0} & =\left[\begin{array}{cc}
0 & 0 \\
0 & \mathbb{1}-\Omega_{\vec{k}} \cdot \Omega_{\vec{k}}^{\dagger}
\end{array}\right], \\
\Omega_{\vec{k}} & =\frac{1}{\sqrt{2 d+2 \cos \vec{k}}}\left[\begin{array}{c}
1+e^{i k_{1}} \\
\vdots \\
1+e^{i k_{d}}
\end{array}\right],
\end{aligned}
$$


with $\cos \vec{k} \equiv \sum_{j=1}^{d} \cos k_{j}$. The eigenvalues of $H^{(\vec{k})}$ are 0 and $\omega^{ \pm}(\vec{k})= \pm \omega(\vec{k})= \pm \sqrt{8 J h(d+\cos \vec{k})}$, from which we can compute the upper bound for the LR speed given by Eq. (31):

$$
v_{\text {Ising }} \leq 2 X_{0} \sqrt{d J h},
$$

where the constant $X_{y}$ is defined as the solution to the equation $x \operatorname{arcsinh}(x)=\sqrt{x^{2}+1}+y$, and $X_{0} \approx 1.50888$. The previous best bound for $v_{\text {Ising }}$ is $8 e d J \approx 21.7 d J$ obtained from the method in Ref. [2], so our bound in Eq. (49) is a major, parametric improvement when $J$ or $d$ is large. Here we emphasize that this is the first LR bound whose speed scales sublinearly with spatial dimension $v \propto$ $\sqrt{d}$-all previously known LR bounds have velocities that scale linearly with $d$ [67]. Even for $J=h, d=2$, Eq. (49) represents a tenfold improvement over the previous bound. See Table I for a summary of the comparison. For the case when $h$ is large, an extension of our method is used to derive a bound that also improves the previous results there as well, as shown in Sec. VIB.

The LR bound for arbitrary local operators can be obtained from Eqs. (16) and (12). For example, for $\left\|\left[\hat{\sigma}_{\vec{r}}^{x}(t), \hat{\sigma}_{\overrightarrow{0}}^{z}(0)\right]\right\|$ we have

$$
\left\|\left[\hat{\sigma}_{\vec{r}}^{x}(t), \hat{\sigma}_{\overrightarrow{0}}^{z}(0)\right]\right\| \leq G_{0,0}(\vec{r}, t)=\int_{-\pi}^{\pi} \cosh [\omega(\vec{k}) t] e^{i \vec{k} \cdot \vec{r}} \frac{d^{d} k}{(2 \pi)^{d}} .
$$

Finally, $\left\|\left[\hat{\sigma}_{\vec{r}}^{x}(t), \hat{\sigma}_{\overrightarrow{0}}^{z}(0)\right]\right\|$ also has a simple LR bound in the form of Eq. (42). Notice that in this case $d_{X Y}$ is the distance between points $X=\vec{r}$ and $Y=\overrightarrow{0}$ on the commutativity graph, which is exactly $2|\vec{r}|$. Therefore, the parameter $u$ needed to satisfy Eq. (38) can be chosen as $u=2 v_{\text {Ising }}$. Inserting Eq. (35) into Eq. (50) we get the simple LR bound

$$
\left\|\left[\hat{\sigma}_{\vec{r}}^{x}(t), \hat{\sigma}_{\overrightarrow{0}}^{z}(0)\right]\right\| \leq C\left(\frac{|\vec{r}|}{v_{\text {Ising }} t}\right)^{-2|\vec{r}|},
$$

where $C$ is a constant.

\section{Extending to arbitrary spin}

The $d$-dimensional spin- $S$ TFIM is defined by the Hamiltonian

$$
\hat{H}=-4 J \sum_{\langle i j\rangle} \hat{S}_{i}^{z} \hat{S}_{j}^{z}-2 h \sum_{j} \hat{S}_{j}^{x}
$$

where $\hat{S}^{\alpha}$ is the spin- $S$ operator satisfying $\left[\hat{S}^{\alpha}, \hat{S}^{\beta}\right]=$ $i \sum_{\gamma} \epsilon^{\alpha \beta \gamma} \hat{S}^{\gamma}, \alpha, \beta, \gamma \in\{x, y, z\}$. Equation (52) is a direct generalization of the spin-1/2 case Eq. (44), so in this case we can simply set $\hat{\gamma}_{\vec{r}, j}=\hat{S}_{\vec{r}}^{z} \hat{S}_{\vec{r}+\hat{e}_{j}}^{z} / S^{2}$ and $\hat{\gamma}_{r, 0}=\hat{S}_{\vec{r}}^{x} / S$, so that the commutativity graph for $\hat{\gamma}_{r, j}, \hat{\gamma}_{r, 0}$ is the same as in Fig. 3, with $J, h$ rescaled to $4 J S^{2}, 2 h S$, respectively. In this way we can borrow the result from the spin-1/2 case, Eq. (49), to get $v_{\mathrm{LR}} \leq 2 X_{0} \sqrt{d J h}(2 S)^{3 / 2}$. This is already qualitatively tighter than the previous best bound $v_{\mathrm{LR}} \leq$ $8 e d J(2 S)^{2}$.

But we can even do much better than this. Recall from the discussion at the end of Sec. II B that the resulting LR bound is typically tightest if we decompose $\hat{H}$ into a form where any two terms either commute or anticommute. Now the difficulty is that in the spin- $S$ case the spin operators $\hat{S}^{x}, \hat{S}^{z}$ neither commute nor anticommute. To overcome this, we can decompose each spin- $S$ operator as a sum of spin-1/2 Pauli operators:

$$
\hat{S}^{\alpha}=\frac{1}{2} \sum_{a=1}^{2 S} \hat{\sigma}_{a}^{\alpha}, \quad \alpha=x, y, z,
$$

by standard addition of angular momentum. The physical Hilbert space on each site is spanned by all the eigenstates of the operator $\left(\hat{S}^{x}\right)^{2}+\left(\hat{S}^{y}\right)^{2}+\left(\hat{S}^{z}\right)^{2}$ with eigenvalue $S(S+1)$, which are simply the states that are fully symmetric under permutation of indices $a$. In the following we derive a LR bound for operators in the enlarged Hilbert space, which automatically gives a bound on operators acting on the physical Hilbert space, since the physical operators (e.g., $\hat{H}, \hat{S}_{j}^{\alpha}$ ) do not couple physical states to unphysical states.

Inserting Eq. (53) into Eq. (52), the Hamiltonian in the enlarged space reads

$$
\hat{H}=-J \sum_{\langle i j\rangle, 1 \leq a, b \leq 2 S} \hat{Z}_{i j}^{a b}-h \sum_{j, 1 \leq a \leq 2 S} \hat{X}_{j}^{a}
$$

where $\hat{Z}_{i j}^{a b} \equiv \hat{\sigma}_{i, a}^{z} \hat{\sigma}_{j, b}^{z}, \hat{X}_{j}^{a} \equiv \hat{\sigma}_{j, a}^{x}$. The commutativity graph of the enlarged Hamiltonian in Eq. (54) can be regarded as a decorated version of the spin-1/2 case shown in Fig. 3, where each triangle is replaced by a set of $2 S$ triangles and each circle is replaced by a set of $\left(\begin{array}{c}2 S \\ 2\end{array}\right)$ circles, each of them linked to a distinct pair of two neighboring triangles, as shown in Fig. 4. In this case Eq. (10) becomes

$$
\begin{aligned}
\dot{\bar{Z}}_{i j}^{a b} & =2 h\left(\bar{X}_{i}^{a}+\bar{X}_{j}^{b}\right), \\
\dot{\bar{X}}_{i}^{a} & =2 J \sum_{j:\langle i j\rangle, 1 \leq b \leq 2 S} \bar{Z}_{i j}^{a b} .
\end{aligned}
$$

Let $X_{i} \equiv \sum_{1 \leq a \leq 2 S} \bar{X}_{i}^{a}$ and $Z_{i j} \equiv \sum_{1 \leq a, b \leq 2 S} \bar{Z}_{i j}^{a b}$, we then have

$$
\begin{aligned}
\dot{\bar{Z}}_{i j} & =4 \operatorname{Sh}\left(\bar{X}_{i}+\bar{X}_{j}\right), \\
\dot{\bar{X}}_{i} & =2 J \sum_{j:\langle i j\rangle} \bar{Z}_{i j} .
\end{aligned}
$$




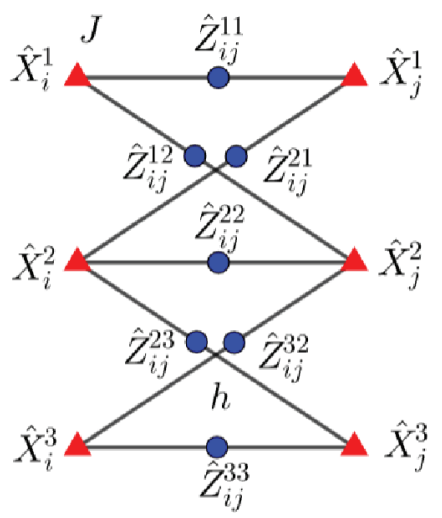

FIG. 4. Commutativity graph $G$ of the spin- $S$ TFIM for the $S=3 / 2$ case. Only two neighboring lattice sites are drawn here.

This is exactly the same as the spin- $1 / 2$ case with substitution $h \rightarrow 2 h S$. Therefore, borrowing the result in Eq. (49), we have

$$
v_{\text {Ising }}^{S} \leq 2 X_{0} \sqrt{2 d J h S},
$$

growing as $\sqrt{S}$ at large $S$, a remarkable improvement over the previous bound, which grows quadratically in $S$. Notice also that if we take the limit $S \rightarrow \infty$ at which $J S$ stays constant (the classical limit), then our LR speed stays finite while the previous bound diverges linearly in $S$.

\section{B. The spin- $S$ Heisenberg $X Y Z$ model} is

The Hamiltonian for the spin- $S$ Heisenberg $X Y Z$ model

$$
\hat{H}=\frac{2}{S} \sum_{\substack{\langle i j\rangle, \alpha=x, y, z}} J_{\alpha} \hat{S}_{i}^{\alpha} \hat{S}_{j}^{\alpha}
$$

where the coefficient is normalized so that the excitation spectrum has a well-defined large- $S$ limit [68]. To get the tightest possible LR bound, we use the same trick as in Sec. V A 2, decomposing each spin- $S$ operator as a sum of spin-1/2 Pauli operators as done in Eq. (53). The enlarged Hamiltonian reads

$$
\hat{H}=\frac{1}{2 S} \sum_{\substack{\langle i j\rangle, 1 \leq a<b \leq 2 S}}\left(J_{x} \hat{X}_{i j}^{a b}+J_{y} \hat{Y}_{i j}^{a b}+J_{z} \hat{Z}_{i j}^{a b}\right),
$$

where $\hat{X}_{i j}^{a b} \equiv \hat{\sigma}_{i a}^{x} \hat{\sigma}_{j b}^{x}$, and similarly for $\hat{Y}_{i j}^{a b}, \hat{Z}_{i j}^{a b}$. The LR bounds for the norm of the commutators $\left[\hat{X}_{i j}^{a b}(t), \hat{B}\right]$, $\left[\hat{Y}_{i j}^{a b}(t), \hat{B}\right]$, and $\left[\hat{Z}_{i j}^{a b}(t), \hat{B}\right]$ for an arbitrary local operator $\hat{B}$ are obtained from the solution to the differential Eq. (10), which in the current case becomes (we omit the superscript
$B$ for notational simplicity)

$$
\begin{aligned}
S \dot{X}_{i j}^{a b}= & \sum_{c \neq b}\left(J_{y} Y_{i j}^{a c}+J_{z} Z_{i j}^{a c}\right)+\sum_{c \neq a}\left(J_{y} Y_{i j}^{c b}+J_{z} Z_{i j}^{c b}\right) \\
& +\sum_{\substack{c, l \neq j: \\
\langle i l\rangle}}\left(J_{y} Y_{i l}^{a c}+J_{z} Z_{i l}^{a c}\right)+\sum_{\substack{c, l \neq i: \\
\langle l j\rangle}}\left(J_{y} Y_{l j}^{c b}+J_{z} Z_{l j}^{c b}\right),
\end{aligned}
$$

where it is assumed that $i, j$ are neighboring sites, and there are two other equations obtained by permuting $X, Y, Z$. The initial condition is similar to Eq. (11). Summing over indices $a, b$, we get

$$
\dot{\mathbf{X}}_{i j}=2(2-1 / S) \mathbf{J} \cdot \mathbf{X}_{i j}+2 \sum_{\substack{l \neq j: \\\langle i l\rangle}} \mathbf{J} \cdot \mathbf{X}_{i l}+2 \sum_{\substack{l \neq i: \\\langle l j}} \mathbf{J} \cdot \mathbf{X}_{l j},
$$

where we use the notation

$$
\mathbf{J}=\left(\begin{array}{ccc}
0 & J_{y} & J_{z} \\
J_{x} & 0 & J_{z} \\
J_{x} & J_{y} & 0
\end{array}\right), \quad \mathbf{X}_{i j}=\sum_{a, b}\left(\begin{array}{c}
X_{i j}^{a b} \\
Y_{i j}^{a b} \\
Z_{i j}^{a b}
\end{array}\right) .
$$

The largest eigenfrequency in a $d$-dimensional hypercubic lattice is $\omega_{m}(\vec{k})=4 J_{m}[\cos \vec{k}+d-1 /(2 S)]$, where $J_{m}=\|\mathbf{J}\|$, so

$$
v_{X Y Z}^{S} \leq 4 d J_{m} X_{1-1 /(2 S d)} .
$$

We now make a comparison with previous best bound and the exact solution in the $S \rightarrow \infty$ limit. For simplicity we focus on the isotropic case $J_{x}=J_{y}=J_{z}=J$. We have $J_{m}=2 J$, so our LR speed remains finite $v_{\mathrm{LR}} \leq 8 d J X_{1}$ in the limit $S \rightarrow \infty$, while the previous bound $v_{\mathrm{LR}} \leq$ $8 d e\left\|\hat{h}_{i j}\right\|=16 \operatorname{deJ}(S+1)$ diverges linearly. Our bound is qualitatively tight in the sense that in the large- $S$ limit, it is only a finite factor of $2 X_{1} \approx 4.47$ bigger than the exact speed $4 d J$ (the spin-wave group velocity), as calculated from the mean-field dispersion relation in Ref. [68]. We emphasize that this is one of the few known examples of a finite LR bound for a system with an infinite local Hilbert space $[54,57,69]$.

\section{Truncated BH model}

We now consider a spin- $S X Y$ model with an additional $\left(S+S_{j}^{z}\right)^{2}$ interaction at each site:

$$
\hat{H}=-\frac{J}{2 S} \sum_{\langle i j\rangle}\left(\hat{S}_{i}^{+} \hat{S}_{j}^{-}+\text {H.c. }\right)+U \sum_{j}\left(S+\hat{S}_{j}^{z}\right)^{2},
$$


where $\hat{S}^{ \pm}=\hat{S}^{x} \pm i \hat{S}^{y}$. At each site $j$ there are $2 S+1$ states $\{|m-S\rangle\}_{m=0}^{2 S}$, and the action of operators $\hat{S}^{ \pm}$is given by

$$
\hat{S}^{ \pm}|m-S\rangle=\left\{\begin{array}{l}
\sqrt{(m+1)(2 S-m)}|m+1-S\rangle \\
\sqrt{m(2 S-m+1)}|m-1-S\rangle
\end{array},\right.
$$

therefore, in the limit $S \rightarrow \infty$ the Hamiltonian in Eq. (65) reproduces the $\mathrm{BH}$ Hamiltonian

$$
\hat{H}=-J \sum_{\langle i j\rangle}\left(\hat{b}_{i}^{\dagger} \hat{b}_{j}+\text { H.c. }\right)+U \sum_{j} \hat{n}_{j}^{2},
$$

if we identify the mapping

$$
\hat{S}^{+} \rightarrow \sqrt{2 S} \hat{b}^{\dagger}, \quad S+\hat{S}^{z} \rightarrow \hat{n}, \quad|m-S\rangle \rightarrow|m\rangle .
$$

In the following we derive a LR bound for the truncated system in Eq. (65). Using the same trick as in Sec. VB, the analog of Eq. (61) is (notice that the term linear in $\hat{S}^{z}$ commutes with $\hat{H}$ and can, therefore, be dropped)

$$
\begin{aligned}
\dot{X}_{i j}^{a b}= & \sum_{c \neq b}\left(\frac{J}{2 S} Y_{i j}^{a c}+U Z_{j}^{b c}\right)+\sum_{c \neq a}\left(\frac{J}{2 S} Y_{i j}^{c b}+U Z_{i}^{a c}\right) \\
& +\sum_{\substack{c, l \neq j: \\
\langle i l\rangle}} \frac{J}{2 S} Y_{i l}^{a c}+\sum_{\substack{c, l \neq i: \\
\langle l j\rangle}} \frac{J}{2 S} Y_{l j}^{c b}, \\
\dot{Z}_{i}^{a b}= & \frac{J}{2 S} \sum_{c, j:\langle j i\rangle}\left(X_{i j}^{a c}+Y_{i j}^{a c}+X_{i j}^{b c}+Y_{i j}^{b c}\right),
\end{aligned}
$$

and another equation similar to the first one with $X$ and $Y$ exchanged. Summing over $a, b$ and doing a Fourier transform, we get

$$
\frac{d}{d t}\left[\begin{array}{c}
X_{\vec{k}} \\
Z_{\vec{k}}
\end{array}\right]=\left[\begin{array}{cc}
2 J\left(d+\cos \vec{k}-\frac{1}{2 S}\right) & 2 S U \\
8 J\left(1-\frac{1}{2 S}\right)(d+\cos \vec{k}) & 0
\end{array}\right]\left[\begin{array}{c}
X_{\vec{k}} \\
Z_{\vec{k}}
\end{array}\right],
$$

where $X_{\vec{k}}$ is the Fourier transform of $X_{i} \equiv \sum_{j:\langle j i, a, b} X_{i j}^{a b}$, and $Z_{\vec{k}}$ is the Fourier transform of $Z_{i} \equiv \sum_{a \neq b} Z_{i}^{a b}$. At large $S$, we have $\omega_{m}(i \vec{\kappa}) \approx 4 \sqrt{2 S U J d} \cosh (\kappa / 2)$, so

$$
v_{\mathrm{LR}} \approx 2 X_{0} \sqrt{2 S U J d} \propto \sqrt{S},
$$

qualitatively tighter than the bound from previous methods, which diverges linearly in $S$. It is known that the actual speed of information propagation in the $S \rightarrow \infty$ limit also diverges [70], and mean-field approximation predicts that the BH model has $v \propto \sqrt{N}$ for an initial coherent state with average occupation number $N$ [71], which suggests that the asymptotic behavior of our bound $v \propto \sqrt{S}$ is tight.

\section{SU(N) FH model}

We next take the $\mathrm{SU}(N) \mathrm{FH}$ model as an example of interacting fermions, using the method in Sec. II C. The Hamiltonian in a general lattice is

$$
\begin{aligned}
\hat{H}= & J \sum_{\langle j l, 1 \leq \sigma \leq N}\left(\hat{a}_{j \sigma}^{\dagger} \hat{a}_{l \sigma}+\text { H.c. }\right) \\
& +\frac{U}{4} \sum_{j, 1 \leq \sigma<\sigma^{\prime} \leq N}\left(2 \hat{n}_{j \sigma}-1\right)\left(2 \hat{n}_{j \sigma^{\prime}}-1\right) .
\end{aligned}
$$

For $N=2$, this is the usual FH model. In the following, we limit our discussion to a bipartite lattice. We first split the fermion creation and annihilation operators to Majorana operators

$$
\hat{a}_{j \sigma}^{\dagger}=i^{(j \in E)} \frac{\hat{c}_{j \sigma}-i \hat{c}_{j \bar{\sigma}}}{2},
$$

where $E$ is the set of even sites, $\bar{\sigma}=-\sigma$, and the Majorana operators satisfy Eq. (20). The Hamiltonian in the Majorana representation is

$$
\begin{aligned}
\hat{H}= & \frac{J}{2} \sum_{\substack{\langle j l\rangle, j \in E, 1 \leq \sigma \leq N}}\left(i \hat{c}_{j \sigma} \hat{c}_{l \sigma}+i \hat{c}_{j \bar{\sigma}} \hat{c}_{l \bar{\sigma}}\right) \\
& -\frac{U}{4} \sum_{j, 1 \leq \sigma<\sigma^{\prime} \leq N} \hat{c}_{j \sigma} \hat{c}_{j \bar{\sigma}} \hat{c}_{j \sigma^{\prime}} \hat{c}_{j \bar{\sigma}^{\prime}} .
\end{aligned}
$$

The norm of the operator $\hat{c}_{j \sigma ; l \sigma^{\prime}}(t) \equiv\left\{\hat{c}_{j \sigma}(t), \hat{c}_{l \sigma^{\prime}}(0)\right\}$ is upper bounded by the solution to Eq. (24), which in the current case becomes (for notational simplicity we omit the labels $l, \sigma^{\prime}$ )

$$
\dot{c}_{j \sigma}=J \sum_{l:\langle j l\rangle} c_{l \sigma}+\frac{U}{2}\left[c_{j \bar{\sigma}}+\sum_{\sigma^{\prime} \neq \sigma}\left(c_{j \sigma^{\prime}}+c_{j \bar{\sigma}^{\prime}}\right)\right],
$$

with initial condition $c_{j \sigma}(0)=2 \delta_{j l} \delta_{\sigma \sigma^{\prime}}$. Equation (74) can be easily solved by a Fourier transform. In a $d$-dimensional hypercubic lattice, we obtain the LR bound

$$
\sum_{1 \leq|\sigma| \leq N}\left\|\left\{\hat{c}_{j \sigma}(t), \hat{c}_{l \sigma^{\prime}}(0)\right\}\right\| \leq 2 e^{[(2 N-1) / 2] U t} I_{\vec{x}_{j}-\vec{x}_{l}}(2 J t),
$$

where $I_{\alpha}(t)$ is the modified Bessel function of the first kind, and $I_{\vec{\alpha}}(t) \equiv \prod_{m=1}^{d} I_{\alpha_{m}}(t)$. The LR speed $v_{\mathrm{FH}}$ can be calculated from Eq. (31) with $\omega_{m}(\vec{k})=2 J \cos \vec{k}+(N-1 / 2) U$, which gives

$$
v_{\mathrm{FH}} \leq 2 X_{(2 N-1) U / 4 d J} d J .
$$

At small $U / J$, this is $3.02 d J$, significantly improving the previous best bound $8 e d J N \approx 21.7 d J N$ [2]. 
At large $N$, the above LR speed scales like $N / \ln N$, which is already qualitatively tighter than the previous bound. But we can do even better than this, to prove a LR speed that grows like $\sqrt{N}$. This can either be achieved by using the commutativity graph method, or by using the fermion method with a slightly different treatment. In the following we take the latter approach for simplicity.

We go back to Eq. (73) and write down the Heisenberg equation for the operators $\hat{c}_{j \sigma}$ and $\hat{u}_{j \sigma \sigma^{\prime}} \equiv \hat{c}_{j \sigma} \hat{c}_{j \sigma} \hat{c}_{j \sigma^{\prime}} \hat{c}_{j \bar{\sigma}^{\prime}}$

$$
\begin{aligned}
\frac{d}{d t} \hat{c}_{j \sigma}= & J \sum_{l:\langle j l\rangle} p_{j} \hat{c}_{l \sigma}+\frac{U}{4} \sum_{\sigma^{\prime} \neq \sigma}\left[\hat{c}_{j \sigma}, \hat{u}_{j \sigma \sigma^{\prime}}\right], \\
\frac{d}{d t} \hat{u}_{j \sigma \sigma^{\prime}}= & \frac{J}{2} \sum_{l:\langle j l\rangle} p_{j} \\
& \times\left[\hat{u}_{j \sigma \sigma^{\prime}}, \hat{c}_{j \sigma} \hat{c}_{l \sigma}+\hat{c}_{j \sigma^{\prime}} \hat{c}_{l \sigma^{\prime}}+\hat{c}_{j \bar{\sigma}} \hat{c}_{l \bar{\sigma}}+\hat{c}_{j \bar{\sigma}^{\prime}} \hat{c}_{l \bar{\sigma}^{\prime}}\right],
\end{aligned}
$$

where $p_{j}=(-1)^{(j \in E)}$. Following the derivations in Eqs. (6)-(11), one can show that the norm of the operators $\left\{\hat{c}_{j \sigma}(t), \hat{c}_{l \tau}(0)\right\},\left[\hat{u}_{j \sigma \sigma^{\prime}}, \hat{c}_{l \tau}(0)\right]$ are upper bounded by the solution to the differential equations

$$
\begin{aligned}
\frac{d}{d t} c_{j \sigma}= & J \sum_{l:\langle j l\rangle} c_{l \sigma}+\frac{U}{2} \sum_{\sigma^{\prime} \neq \sigma} u_{j \sigma \sigma^{\prime}}, \\
\frac{d}{d t} u_{j \sigma \sigma^{\prime}}= & J \sum_{l:\langle j l\rangle}\left[c_{j \sigma}+c_{l \sigma}+c_{j \sigma^{\prime}}+c_{l \sigma^{\prime}}\right. \\
& \left.+\left(\sigma, \sigma^{\prime} \rightarrow \bar{\sigma}, \bar{\sigma}^{\prime}\right)\right],
\end{aligned}
$$

with initial condition $c_{j \sigma}(0)=2 \delta_{j l} \delta_{\sigma \tau}, u_{j \sigma \sigma^{\prime}}(0)=2 \delta_{j l}(\tau \in$ $\left.\left\{\sigma, \bar{\sigma}, \sigma^{\prime}, \bar{\sigma}^{\prime}\right\}\right)$. The largest eigenfrequency is

$\omega^{ \pm}(\vec{k})=J \cos \vec{k} \pm \sqrt{J^{2} \cos ^{2} \vec{k}+4 U J(N-1)(d+\cos \vec{k})}$,

and the LR speed is again computed from Eq. (31). The result is $v_{\mathrm{FH}} \leq Z_{(N-1) d U / J} J$, where

$$
Z_{y} \equiv \min _{\kappa>0} \frac{\cosh \kappa+\sqrt{\cosh ^{2} \kappa+4 y(1+\cosh \kappa)}}{\kappa} .
$$

At large $y, Z_{y} \approx X_{0} \sqrt{2 y}$, so

$$
v_{\mathrm{FH}} \approx X_{0} \sqrt{2 N d U J}, \quad \text { for large } N,
$$

a significant qualitative improvement over the previous linear growth. In the classical limit $N \rightarrow \infty$ where $\left\langle\hat{n}_{j}\right\rangle / N$ and $U N$ stays constant, our LR speed remains finite while the previous bound diverges linearly in $N$.

For the 1D SU(2) case, the comparison between the LR speeds calculated from different methods is shown in

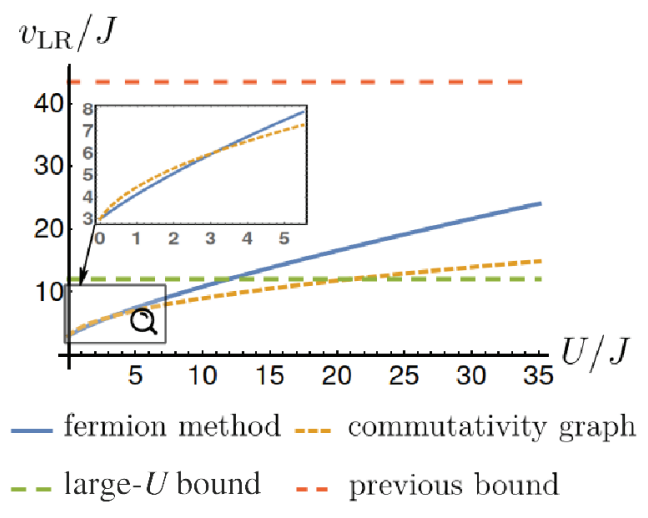

FIG. 5. Comparison between different upper bounds for the LR speed of the 1D FH model, using the fermion method, the commutativity graph, and the large- $U$ method, Eqs. (24), (10), and (95), respectively. The previous best bound is $16 \mathrm{eJ}$ [2].

Fig. 5. Both methods introduced so far in this section substantially improve the previous bound for $U / J \leq 80$. Results that also improve the previous bound at large $U$ are derived in Sec. VIC.

In the derivations above we did not use the commutativity graph method, but the method in Sec. IV can still be applied to derive the simple LR bound, with $d_{j l}=\left|\vec{x}_{j}-\vec{x}_{l}\right|$, and $u=\min \left\{2 X_{(2 N-1) U / 4 d J} d J, Z_{(N-1) d U / J} J\right\}$, and we have

$$
\left\|\left\{\hat{c}_{j \sigma}(t), \hat{c}_{l \sigma^{\prime}}(0)\right\}\right\| \leq C\left(\frac{u t}{d_{j l}}\right)^{d_{j l}},
$$

for $\forall j, l, \sigma, \sigma^{\prime}$, where $C$ is a constant.

We now compare the above bounds with the exact unequal time anticommutator at the noninteracting point $U=0$ in a $d$-dimensional square lattice, to investigate how tight these bounds are. The Hamiltonian is diagonalized as

$$
\hat{H}=\int_{\mathrm{BZ}} \omega(\vec{k}) \sum_{1 \leq \sigma \leq N} \hat{a}_{\vec{k} \sigma}^{\dagger} \hat{a}_{\vec{k} \sigma} d^{d} k,
$$

where $\omega(\vec{k})=2 J \cos \vec{k}$. Therefore,

$$
\begin{aligned}
\hat{a}_{i \sigma}(t) & =\frac{1}{(2 \pi)^{d / 2}} \int_{-\pi}^{\pi} \hat{a}_{\vec{k} \sigma} e^{-i \omega(\vec{k}) t} e^{i \vec{k} \cdot \vec{x}} d^{d} k \\
& =\sum_{j} J_{\vec{x}_{i}-\vec{x}_{j}}(2 J t) \hat{a}_{j \sigma}(0),
\end{aligned}
$$

where $J_{n}(t)$ is the Bessel function, and we define $J_{\vec{n}}(t) \equiv$ $\prod_{m=1}^{d} J_{n_{m}}(t)$. Inserting Eq. (84) into the lhs of Eq. (75), we get

$$
\sum_{1 \leq \sigma \leq N}\left\|\left\{\hat{c}_{j \sigma}(t), \hat{c}_{l \sigma^{\prime}}(0)\right\}\right\|=2\left|J_{\vec{x}_{j}-\vec{x}_{l}}(2 J t)\right| .
$$

Comparing this with our bound in Eq. (75) [notice that the modified fermion method Eq. (78) also leads to the 
same result when $U=0$ ], we see that at the noninteracting point $U=0$, our LR bound just replaces the Bessel function $J_{\vec{x}_{j}-\vec{x}_{l}}(2 J t)$ of the exact solution by the modified Bessel function $I_{\vec{x}_{j}}-\vec{x}_{l}(2 J t)$. Since both $J_{x}(t)$ and $I_{x}(t)$ have the same asymptotic form $1 / x !(t / 2)^{x}$ when $x / t$ is large, we conclude that our LR bound Eq. (75) has the tightest large- $x$ and small- $t$ exponent at the noninteracting point, where the large- $x$ exponent $\zeta$ of a bound $f(x, t)$ is defined as $\sup \left\{\zeta \mid \lim _{x \rightarrow \infty} e^{\zeta x \ln x} f(x, t)\right.$ exists, $\left.\forall t>0\right\}$. [Our bounds Eqs. (75) and (82) and the exact solution Eq. (85ss) all have $\zeta=1$.]

\section{E. Wen's quantum rotor model}

In this section we consider Wen's quantum rotor model [72-74]. The model is defined on a $d \geq 2$ dimensional hypercubic lattice whose sites are labeled by $\vec{r}$ and edges are labeled by $(\vec{r} \alpha) \equiv\left\langle\vec{r}, \vec{r}+\vec{e}_{\alpha}\right\rangle, 1 \leq \alpha \leq d$. On each edge $(\vec{r} \alpha)$ there is a $2 \mathrm{D}$ quantum rotor, with angle variable $\hat{\theta}_{\vec{r} \alpha}$ and the dual angular momentum $\hat{S}_{\vec{r} \alpha}$, satisfying the canonical quantization condition $\left[\hat{\theta}_{r^{\prime} \alpha}, \hat{S}_{\vec{r}^{\prime} \beta}\right]=i \delta_{\vec{r}, r^{\prime}} \delta_{\alpha \beta}$. The Hamiltonian is

$$
\hat{H}=J \sum_{\vec{r}, 1 \leq \alpha \leq d} \hat{S}_{\vec{r} \alpha}^{2}-\frac{g}{2} \sum_{\vec{r}, \alpha \neq \beta} \hat{W}_{\vec{r} \alpha \beta}
$$

where $\hat{W}_{\vec{r} \alpha \beta}=\exp \left(i \hat{\theta}_{\vec{r} \alpha}+i \hat{\theta}_{\vec{r}+\vec{e}_{\beta}, \alpha}-i \hat{\theta}_{\vec{r} \beta}-i \hat{\theta}_{\vec{r}+\vec{e}_{\alpha}, \beta}\right)$. Notice that $\hat{W}_{\vec{r} \alpha \beta}=\hat{W}_{\vec{r} \beta \alpha}^{\dagger}$.

Based on some semiclassical treatments, Refs. [72-74] have shown that in the topologically ordered phase $J \ll$ $g$, this system hosts bosonic excitations propagating at speed $\sqrt{2 g J}$, behaving like artificial light waves. Ref. [53] studied a three-state truncated version of this model, and proved a LR bound with velocity $e \sqrt{2 g J}$. However, their method would fail in the untruncated version Eq. (86), since the term $\left(\hat{S}_{\vec{r} \alpha}\right)^{2}$ has infinite operator norm $\left\|\hat{S}_{\vec{r} \alpha}^{2}\right\|=S^{2}$ (the number of states on a single site is $2 S+1$ ), leading to a LR speed that diverges linear in $S$. Understanding what happens as $S \rightarrow \infty$ is important, since the argument that light emerges is valid only in this limit. In the following we use a slightly modified version of our method to overcome this difficulty, and prove that $v_{\mathrm{LR}} \leq 2 X_{0} \sqrt{(d-1) g J}$.

We begin by writing down the Heisenberg equations for $\hat{S}_{\vec{r} \alpha}, \hat{W}_{\vec{r} \alpha \beta}$

$$
\begin{aligned}
i \partial_{t} \hat{S}_{\vec{r} \alpha} & =-\frac{g}{2} \sum_{\beta}\left[\hat{W}_{\vec{r} \alpha \beta}+\hat{W}_{\vec{r}-\hat{e}_{\beta}, \alpha \beta}-(\alpha \leftrightarrow \beta)\right], \\
i \partial_{t} \hat{W}_{\vec{r} \alpha \beta} & =J\left\{\hat{W}_{\vec{r} \alpha \beta}, \hat{S}_{\vec{r} \beta}+\hat{S}_{\vec{r}+\vec{e}_{\alpha}, \beta}-(\alpha \leftrightarrow \beta)\right\},
\end{aligned}
$$

where we use $\left[\hat{S}_{\vec{r} \alpha}, e^{ \pm i \hat{\theta}_{r^{\prime}} \beta}\right]= \pm e^{ \pm i \hat{\theta}_{\vec{r}^{\prime}} \beta} \delta_{\vec{r}, \vec{r}^{\prime}} \delta_{\alpha \beta}$. Taking the commutator with an arbitrary local operator $\hat{B}$, we have

$$
\begin{aligned}
i \partial_{t} \hat{S}_{\vec{r} \alpha}^{B}= & -\frac{g}{2} \sum_{\beta}\left[\hat{W}_{\vec{r} \alpha \beta}^{B}+\hat{W}_{\vec{r}-\hat{e}_{\beta}, \alpha \beta}^{B}-(\alpha \leftrightarrow \beta)\right], \\
i \partial_{t} \hat{W}_{\vec{r} \alpha \beta}^{B}= & J\left\{\hat{W}_{\vec{r} \alpha \beta}^{B}, \hat{S}_{\vec{r} \beta}+\hat{S}_{\vec{r}+\vec{e}_{\alpha}, \beta}-(\alpha \leftrightarrow \beta)\right\} \\
& +J\left\{\hat{W}_{\vec{r} \alpha \beta}, \hat{S}_{\vec{r} \beta}^{B}+\hat{S}_{\vec{r}+\vec{e}_{\alpha}, \beta}^{B}-(\alpha \leftrightarrow \beta)\right\} .
\end{aligned}
$$

Now we get rid of the first term in the second equation by doing a change of variable $\hat{W}_{\vec{r} \alpha \beta}^{B}(t)=\hat{U}_{\vec{r} \alpha \beta}(t) \tilde{W}_{\vec{r} \alpha \beta}^{B} \hat{V}_{\vec{r} \alpha \beta}(t)$, where $\quad \hat{U}_{\vec{r} \alpha \beta}(0)=\hat{V}_{\vec{r} \alpha \beta}(0)=\hat{I}, \quad i \partial_{t} \hat{U}_{\vec{r} \alpha \beta}(t)=J\left[\hat{S}_{\vec{r} \beta}(t)+\right.$ $\left.\hat{S}_{\vec{r}+\vec{e}_{\alpha}, \beta}(t)-(\alpha \leftrightarrow \beta)\right] \hat{U}_{\vec{r} \alpha \beta}(t)$, and $i \partial_{t} \hat{V}_{\vec{r} \alpha \beta}(t)=\hat{V}_{\vec{r} \alpha \beta}(t)$ $J\left[\hat{S}_{\vec{r} \beta}(t)+\hat{S}_{\vec{r}+\vec{e}_{\alpha}, \beta}(t)-(\alpha \leftrightarrow \beta)\right]$. Then using the same derivations in Eqs. (7)-(10), we can prove that $\left\|\hat{S}_{\vec{r} \alpha}^{B}\right\|$ and $\left\|\hat{W}_{\vec{r} \alpha \beta}^{B}\right\|$ are upper bounded by the solution to the differential equation:

$$
\begin{aligned}
\dot{S}_{\vec{r} \alpha} & =g \sum_{\beta \neq \alpha}\left(W_{\vec{r} \alpha \beta}+W_{\vec{r}-\vec{e}_{\beta}, \alpha \beta}\right) \\
\dot{W}_{\vec{r} \alpha \beta} & =2 J\left(S_{\vec{r} \alpha}+S_{\vec{r} \beta}+S_{\vec{r}+\vec{e}_{\alpha}, \beta}+S_{\vec{r}+\vec{e}_{\beta}, \alpha}\right),
\end{aligned}
$$

with initial condition $S_{\vec{r} \alpha}(0)=\left\|\hat{S}_{\vec{r} \alpha}^{B}(0)\right\|, W_{\vec{r} \alpha \beta}(0)=$ $\left\|\hat{W}_{\vec{r} \alpha \beta}^{B}(0)\right\|$. The maximal eigenfrequency at $\vec{k}=i \vec{\kappa}$ is $\omega_{m}(i \vec{\kappa})=4 \sqrt{(d-1) g J} \cosh (\kappa / 2)$, therefore, using Eq. (31) we get

$$
v_{\mathrm{LR}} \leq 2 X_{0} \sqrt{(d-1) g J} .
$$

This is our second example (after the large- $S$ Heisenberg $X Y Z$ model) of a finite LR speed in a system with an infinite local Hilbert space, and a second example (after TFIM) of a LR speed that grows sublinearly with spatial dimension $v \propto \sqrt{d}$. It is also a good bound quantitatively - in 2D, it is only larger than the semiclassical result $v=\sqrt{2 g J}$ by a factor of $\sqrt{2} X_{0} \approx 2.1[75]$.

\section{IMPROVING THE LIEB-ROBINSON VELOCITY BY ELIMINATING LARGE COUPLING CONSTANTS}

In several examples discussed in the previous section, our commutativity graph method dramatically improves the LR velocities when the local Hilbert space dimension or spatial dimension becomes large. Most prominently, in the large spin $S \rightarrow \infty$ limit of Heisenberg $X Y Z$ model and Wen's quantum rotor model, our method gives finite LR velocities, which removes the unphysical divergence in previous bounds. Nevertheless, there is still another type of unphysical divergence present in these bounds. This happens when a Hamiltonian is a sum of terms, and one 
set of mutually commuting terms is multiplied by coefficients that become very large. For example, in the large- $J$ or large- $h$ limits of TFIM in Eq. (49), the large- $U$ limit of the SU(N) FH model in Eqs. (76) and (81), and in the large- $J$ or large- $g$ limit of Wen's quantum rotor model for any finite spin $S$, the bounds for the LR velocity diverge, while in reality, the actual velocities of information propagation in these limits are expected to be finite. This kind of divergence renders the LR bound infinitely weak in these limits, a limitation that has plagued prior LR bounds.

We introduce a method, extending the results above, that removes this second type of unphysical divergence and gives a finite LR velocity in these limits. The basic idea is to derive LR bounds from coupled differential equations on a subset of the commutativity graph that has removed the operators associated with large coefficients. This is accomplished by an appropriate unitary transform of the remaining operators. Although similar methods were employed in previous works $[14,40,43]$, they were only used to remove single-site terms, while our method can be used to remove arbitrary mutually commuting terms in the Hamiltonian. In the following we first present the general technique in Sec. VI A, then in Secs. VI B to VI D we apply this technique to the specific examples mentioned above, and finally present a general result in Sec. VIE.

\section{A. Eliminating large coupling constants on the commutativity graph}

Consider the commutativity graph $G=\left(V_{G}, E_{G}\right)$ of a locally interacting spin Hamiltonian defined in Eq. (4), where $V_{G}$ is the set of vertices and $E_{G}$ is the set of edges. Let $F \subset V_{G}$ denote a subset of disjoint vertices of $G$, such that $\left\{h_{j} \mid j \in F\right\}$ is the set of large parameters we want to get rid of. The fact that elements of $F$ are disjoint vertices in $G$ means that the corresponding operators $\left\{\hat{\gamma}_{j} \mid j \in F\right\}$ mutually commute. We now construct a graph $G_{F}^{\prime}=\left(V^{\prime}, E^{\prime}\right)$ from $G$ in the following way: (1) the vertices $V^{\prime}=V_{G} \backslash F$ of $G_{F}^{\prime}$ are obtained from $V_{G}$ by removing elements of $F$; (2) $E^{\prime}$ is defined such that for $\forall i, j \in V^{\prime},\langle i j\rangle \in E^{\prime}$ if and only if either $\langle i j\rangle \in E_{G}$ or there exists an $l \in F$ such that $\langle i l\rangle \in E_{G},\langle l j\rangle \in E_{G}$. In the left (right) panel of Fig. 6 we show the graph $G_{F}^{\prime}$ after removing parameter $J(h)$, respectively.

Let us denote by $\hat{I}$ the sum of all Hamiltonian terms corresponding to vertices in $F$ (i.e., the unwanted terms):

$$
\hat{I}=\sum_{j \in F} h_{j} \hat{\gamma}_{j}
$$

The important step here is to consider the evolution of the operator $\hat{\gamma}_{i}$ (for $i \in G_{F}^{\prime}$ ) in a way that the unwanted term $\hat{I}$ is "rotated away" by a unitary transformation:

$$
\hat{\gamma}_{i}(s, t)=e^{i s \hat{H}} e^{i(t-s) \hat{I}} \hat{\gamma}_{i} e^{-i(t-s) \hat{I}} e^{-i s \hat{H}} .
$$
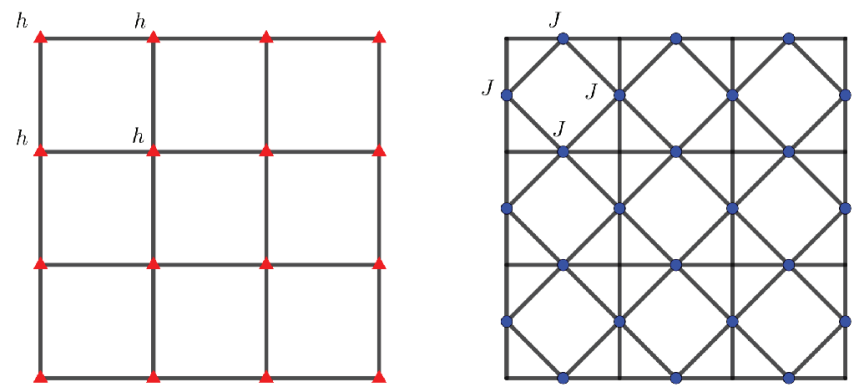

FIG. 6. Graph $G_{F}^{\prime}$ is constructed from the commutativity graph $G$ of the 2D TFIM in Fig. 3 by removing large parameters, by the construction in Sec. VI A. Left: $G_{F}^{\prime}$ for $F$ being the set of vertices representing $\hat{\sigma}_{i}^{z} \hat{\sigma}_{j}^{z}$ terms, i.e., removing parameter $J$. Right: $G_{F}^{\prime}$ for $F$ being the set of vertices representing $\hat{\sigma}_{i}^{x}$ terms, i.e., removing parameter $h$.

Notice that $\hat{\gamma}_{i}(t, t)=\hat{\gamma}_{i}(t)$, the operator in Heisenberg picture. Taking derivative with respect to $s$, we have

$$
\begin{aligned}
\partial_{s} \hat{\gamma}_{i}(s, t) & =e^{i s \hat{H}} i\left[\hat{H}-\hat{I}, \hat{\gamma}_{i}(0, t-s)\right] e^{-i s \hat{H}} \\
& =i \sum_{j \in G_{F}^{\prime},\langle i j\rangle \in G_{F}^{\prime}} h_{j}\left[\hat{\gamma}_{j}(s), \hat{\gamma}_{i}(s, t)\right],
\end{aligned}
$$

where the sum is over all terms in $\hat{H}-\hat{I}$ that do not commute with $\hat{\gamma}_{i}(0, t-s)$. Equation (93) should be considered as the analog of Eq. (5). Now the same derivations as in Eqs. (6)-(8) for the commutator $\hat{\gamma}_{i}^{B}(s, t)=\left[\hat{\gamma}_{i}(s, t), \hat{B}(0)\right]$ result in

$$
\begin{aligned}
\left\|\hat{\gamma}_{i}^{B}(t)\right\| \leq\left\|\hat{\gamma}_{i}^{B}(0, t)\right\|+2 \sum_{\substack{j \in G_{F}^{\prime}: \\
\langle i j\rangle \in G_{F}^{\prime}}}\left|h_{j}\right| \int_{0}^{t}\left\|\hat{\gamma}_{j}^{B}(s)\right\| d s \\
\leq 2\|\hat{B}\|+2 \sum_{\substack{j \in G_{F}^{\prime}: \\
\langle i j\rangle \in G_{F}^{\prime}}}\left|h_{j}\right| \int_{0}^{t}\left\|\hat{\gamma}_{j}^{B}(s)\right\| d s .
\end{aligned}
$$

In this case the generalized Grönwall's inequality [63] indicates that $\left\|\hat{\gamma}_{i}^{B}(t)\right\|$ is upper bounded by the solution $\gamma_{i}^{B}(t)$ to the differential equation

$$
\dot{\gamma}_{i}^{B}(t)=2 \sum_{j \in G_{F}^{\prime}:\langle i j\rangle \in G_{F}^{\prime}}\left|h_{j}\right| \gamma_{j}^{B}(t)
$$

with initial value $\gamma_{i}^{B}(0)=2\|\hat{B}\|$. Equation (95) is the same as Eq. (10) with $G$ replaced by $G_{F}^{\prime}$.

In summary, when the parameters of a set of disjoint vertices of $G$ become very large, we can get a better bound for the speed of information propagation by solving the differential Eq. (95) on the graph $G_{F}^{\prime}$, which has the large parameters removed, and links added when two vertices of $G_{F}^{\prime}$ are both connected to a (removed) vertex in the original 
graph. Then all the methods we introduce in Secs. III and IV apply equally well. The simple form of the bound in Eq. (3) is still true provided that $d_{X Y}$ and $u$ are understood as the distance and LR speed on the graph $G_{F}^{\prime}$, respectively [76].

\section{B. Example: TFIM at large $\boldsymbol{J}$ or large $\boldsymbol{h}$}

As a first example, in the case of the TFIM, the left (right) panel of Fig. 6 shows the graph $G_{F}^{\prime}$ constructed from $G$ after eliminating the parameter $J$ (the parameter $h$ ) for the $d=2$ case, and the resulting upper bound for the LR speed is $4 X_{0} d h\left[4 X_{(d-1) / d} d J\right]$. Therefore, combined with Eq. (49), we have the upper bound for the LR speed

$$
v_{\text {Ising }} \leq \min \left\{2 X_{0} \sqrt{d J h}, 4 X_{(d-1) / d} d J, 4 X_{0} d h\right\}
$$

for the $d$-dimensional TFIM. This is a major improvement compared to the previous best bound $v_{\text {Ising }} \leq 8 e d J$ (calculated from the method in Ref. [2]), as we not only removed unphysical divergence of $v_{\text {Ising }}$ in the large- $J$ limit, but also tightened it by a factor of at least 2.4 for all values of $d$, $J$, and $h$. [Notice, however, that in 1D, a special method is available in a recent paper Ref. [77], which gives a bound for LR speed $2 e J \approx 5.44 J$ for the $1 \mathrm{D}$ TFIM, which is about $10 \%$ tighter than our bound $4 X_{0} J \approx 6.04 J$ in the large- $h$ limit. Away from the large- $h$ limit, when $h / J<$ 3.24, our bound $\min \left\{2 X_{0} \sqrt{J h}, 4 X_{0} h\right\}$ remains the tightest. Furthermore, it is possible to combine their methods and our method of commutativity graph, which will tighten all the three velocity bounds for 1D TFIM in Eq. (96) by roughly $10 \%$. The result is $v_{\mathrm{LR}} \leq \min \{e \sqrt{J h}, 2 e J, 2 e h\}$. Notice also that if we use Theorem 4 of Ref. [77] in our commutativity graph, we may be able to tighten our velocity bounds in higher dimensions as well. However, in $d>1$ it may be a hard combinatorial problem to compute the sum over all irreducible paths in Theorem 4 of Ref. [77], so we leave this as a future direction.]

\section{Example: $\mathrm{SU}(\boldsymbol{N}) \mathrm{FH}$ model at large $\boldsymbol{U}$}

As a second example, we consider the $\mathrm{SU}(N)$ FH model. Here, the tightest large- $U$ bound is calculated from the commutativity graph $G_{F}^{\prime}$, which is constructed from $G$ in Fig. 7 by eliminating the interaction term (which is $\propto U$ ) according to the prescription of this section. The result for the LR speed is $v_{\mathrm{FH}} \leq 4 X_{0} N J$. Combined with the results of Sec. V D, we have the upper bound

$$
v_{\mathrm{FH}} \leq \min \left\{2 X_{(2 N-1) U / 4 J} J, Z_{(N-1) U / J} J, 4 X_{0} N J\right\}
$$

for the 1D $\mathrm{SU}(N) \mathrm{FH}$ model, which is also a significant improvement of the previous best bound $v_{\mathrm{FH}} \leq 8 e N J$, see Fig. 5 for the comparison.
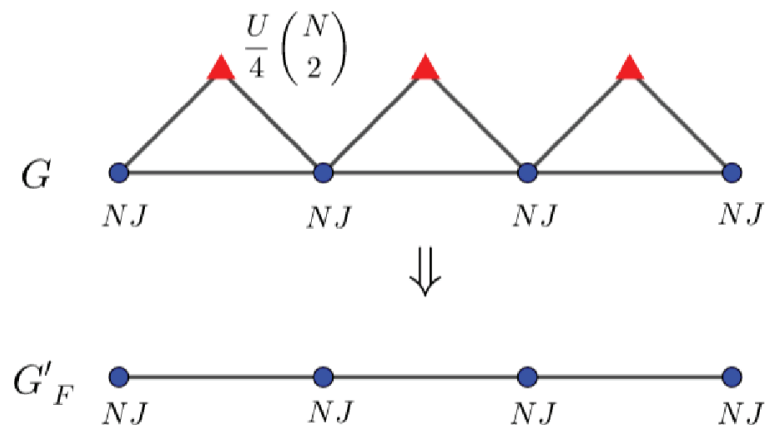

FIG. 7. Commutativity graph $G$ and the reduced graph $G_{F}^{\prime}$ of the $1 \mathrm{D} \mathrm{SU}(N) \mathrm{FH}$ model. Here the blue circles represent the term $\sum_{\sigma} i \hat{c}_{j \sigma} \hat{c}_{j+1, \sigma}$ while the red triangles represent $\sum_{1 \leq \sigma<\sigma^{\prime} \leq N} \hat{c}_{j \sigma} \hat{c}_{j \bar{\sigma}} \hat{c}_{j \sigma^{\prime}} \hat{c}_{j \bar{\sigma}^{\prime}} \cdot G_{F}^{\prime}$ is obtained from $G$ by removing the red triangles.

\section{Example: Wen's quantum rotor model at large $J$ or large $g$}

As a third example of eliminating large parameters, we consider the spin- $S$ truncated version of Wen's quantum rotor model, whose Hamiltonian has the same form as Eq. (86), with $\hat{S}, e^{i \hat{\theta}}$ (on each site) replaced by the following $(2 S+1) \times(2 S+1)$ matrices:

$$
\hat{S}=\operatorname{diag}\{S, S-1, \ldots,-S\}, \quad e^{i \hat{\theta}}=\left(\begin{array}{cc}
0 & I_{2 S} \\
1 & 0
\end{array}\right),
$$

where $I_{2 S}$ is the $2 S \times 2 S$ identity matrix. Ref. [53] studied the $2 \mathrm{D} S=1$ case, and proved a LR bound with velocity $e \sqrt{2 g J}$, in the topologically ordered phase $J \ll g$. Here we prove that in this limit $J \ll g$ the LR speed actually has a much tighter upper bound $v \leq$ const. $\times J S^{2}$. For illustrative purpose, we focus on the $2 \mathrm{D}$ case for simplicity, but the generalization to arbitrary spatial dimension is straightforward. To prove this, we first notice that the commutativity graph of the Hamiltonian terms $\hat{S}_{\vec{r} \alpha}^{2}-S^{2} / 2$ and $\hat{W}_{\vec{r} \alpha \beta}+$ H.c. is exactly the same with that of 2D TFIM shown in Fig. 3, where the blue circles represent the $S$ terms, with parameter $J S^{2} / 2$, and the red triangles represent the $W$ terms, with parameter $g$. Therefore, we can borrow the result from the TFIM with substitutions $J \rightarrow$ $J S^{2} / 2, h \rightarrow g$, and we get $v_{\mathrm{LR}} \leq \min \left\{4 X_{1 / 2} J S^{2}, 8 X_{0} g\right\}$, which is much tighter than the result of Ref. [53] $e \sqrt{2 g J} S$ in the small $J$ limit for any finite $S$. Combined with the result of Sec. VE, we obtain

$$
v_{\text {rotor }} \leq \min \left\{4 X_{1 / 2} J S^{2}, 2 X_{0} \sqrt{(d-1) g J}, 8 X_{0} g\right\} .
$$

Our bound indicates that the semiclassical treatment of the rotor model would fail for any finite $S$ for sufficiently small $J$ (with fixed $g$ ), since the semiclassical approximation predicts emergent light traveling at speed $\sqrt{2 g J}$, which violates our bound for sufficiently small $J$. In the 
$S \rightarrow \infty$ limit, however, our large- $J$ bound diverges, and the method in Sec. VE has to be used, which gives $v \leq$ const. $\sqrt{g J}$, agreeing with the semiclassical result.

\section{E. A general result on perturbed solvable models with mutually commuting terms}

We end this section by making a general statement about a family of perturbed exactly solvable models, whose Hamiltonian has the form

$$
\hat{H}=\hat{H}_{0}+J \sum_{j} \hat{V}_{j}
$$

where $\hat{H}_{0}$ is the solvable part and $J \sum_{j} \hat{V}_{j}$ is a sum of local perturbations. Here $j$ is a label for terms in the Hamiltonian (it does not necessarily mean a single site: $\hat{V}_{j}$ can include multibody interactions). We make the following assumptions:

(1) $\hat{H}_{0}=\sum_{i} \hat{h}_{i}$ is the sum of mutually commuting, locally interacting terms, i.e., $\left[h_{i}, h_{j}\right]=0, \forall i, j$. Primary examples include the $J$ term or the $h$ term of TFIM, the interaction term of $\mathrm{BH}$ model or $\mathrm{SU}(N)$ FH model, the $J$ term or the $g$ term of the spin- $S$ truncated Wen's quantum rotor model, the stringnet models [62] with Kitaev's toric code model [61] being a prominent special case, and the exactly solvable fracton models [78-80].

(2) There is a uniform upper bound for the norm of local perturbations $\left\|V_{j}\right\| \leq c, \forall j$. This is true for any translation invariant system with a finite local Hilbert space.

Then we have the following.

Theorem 1. There exists constant $c^{\prime}>0$ such that $v_{\mathrm{LR}} \leq$ $c^{\prime}|J|$, i.e., the LR speed of the model Eq. (100) grows at most linearly in the strength of the perturbation.

Notice that the second condition is necessary, since in Wen's quantum rotor model with infinite $S$, in the limit $J \ll g$, the semiclassical treatment gives artificial light propagating at speed $v \propto \sqrt{J g}$, which does not satisfy $v \leq c^{\prime}|J|$ for sufficiently small $J$. Theorem 1 can be proved by using the prescription of this section to eliminate all the terms in $\hat{H}_{0}$, the reduced graph $G_{F}^{\prime}$ only involves vertices representing local perturbations $\hat{V}_{i}$. Locality of the original Hamiltonian $\hat{H}$ implies a uniform upper bound on the degree of every vertex in $G_{F}^{\prime}$, which, combined with condition (2), yields a LR bound with $v_{\mathrm{LR}} \leq c^{\prime}|J|$, based on similar treatment as in Eq. (39). The previous bounds for the Hamiltonian Eq. (100) give an LR speed that stays finite in the limit $J \rightarrow 0$ unless $\hat{H}_{0}$ only contains onsite terms (which is not the case for models with topological order), since the previous $v_{\mathrm{LR}}$ is proportional to the operator norm of local Hamiltonians $\hat{h}_{a}$ in the solvable part $\hat{H}_{0}$. Our bound, which vanishes linearly in perturbation strength $J$, is a qualitative improvement.

\section{CONSEQUENCES OF OUR LR BOUNDS ON GROUND-STATE CORRELATION DECAY}

Our tighter LR bounds can immediately translate into improved bounds in all areas that LR bounds are used, such as bounding the timescale for dynamical processes [3,6, 12], studying equilibrium properties like exponential decay of connected correlation functions [13-16,19], quantifying the entanglement area law [24], and providing error bounds on numerical algorithms [31-33,35,36]. In this section we demonstrate this with one of the most important consequences of LR bounds, establishing the decay of ground-state correlations in gapped, locally interacting systems [13-16].

We achieve this goal by directly replacing the previous LR bound by our bound Eq. (3) in the proof of Ref. [14], and obtain an upper bound on the correlation length $\xi$. The bound is not only a quantitative improvement, but are qualitatively tighter in several limits, e.g., in the $J / h \rightarrow \infty$ limit of TFIM and the $S \rightarrow \infty$ limit of Heisenberg $X X Z$, where our bounds have the same asymptotic scaling as the known exact solutions.

Let us start with the following well-established inequality, which relates the ground-state correlation to the unequal time commutator $[2,14]$

$$
\begin{aligned}
\left|\left\langle G\left|\hat{A}_{X} \hat{B}_{Y}\right| G\right\rangle\right| \leq & \frac{1}{\pi} \int_{0}^{\infty} \frac{e^{-\alpha t^{2}}}{|t|}\left\|\left[\hat{A}_{X}(t), \hat{B}_{Y}\right]\right\| d t \\
& +c_{0} e^{-\Delta^{2} / 4 \alpha}, \forall \alpha>0
\end{aligned}
$$

where $c_{0}$ is a constant independent of $\alpha$ and $t$, and we assume $\left\langle G\left|\hat{A}_{X}\right| G\right\rangle=\left\langle G\left|\hat{B}_{Y}\right| G\right\rangle=0$ for simplicity. We split the integral over $t$ into two regions, which we treat differently: for $t<r / u$ we apply our LR bound in Eq. (3), while for $t \geq r / u$ we use the trivial bound $\left\|\left[\hat{A}_{X}(t), \hat{B}_{Y}\right]\right\| \leq$ $2\|\hat{A}\|\|\hat{B}\| \equiv c_{1}$. We get

$$
\begin{aligned}
\left|\left\langle G\left|\hat{A}_{X} \hat{B}_{Y}\right| G\right\rangle\right| \leq & \frac{C}{\pi} \int_{0}^{r / u} \frac{e^{-\alpha t^{2}}}{t}\left(\frac{u t}{r}\right)^{r} d t \\
& +\frac{c_{1}}{\pi} \int_{r / u}^{\infty} \frac{e^{-\alpha t^{2}}}{t} d t+c_{0} e^{-\Delta^{2} / 4 \alpha} \\
\leq & \frac{C}{\pi} \int_{0}^{1 / u} \frac{e^{-\alpha r^{2} \tau^{2}}}{\tau}(u \tau)^{r} d \tau \\
& +\frac{c_{1}}{\pi} \frac{e^{-\alpha r^{2} / u^{2}}}{2 \alpha r^{2} / u^{2}}+c_{0} e^{-\Delta^{2} / 4 \alpha} .
\end{aligned}
$$




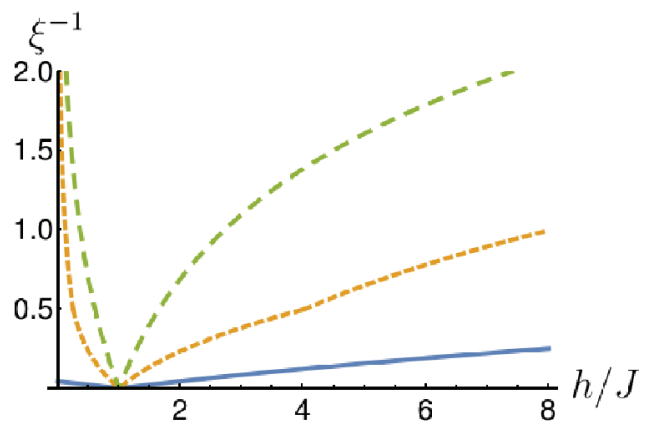

- previous --- current - - exact

FIG. 8. Comparison of the lower bounds for $\xi^{-1}$ in the 1D TFIM, between the result of Ref. [14] and the current paper, and the exact solution given in Ref. [81]. In the limit $h \gg J$, all three curves scale as $\ln (h / J)$, while in the limit $h \ll J$, our bound and the exact solution scale as $\ln (J / h)$, while the previous bound approaches a constant 0.065 .

where $r=d_{X Y}, C$ is a constant defined in Eq. (42), and in the second step we apply the inequality $e^{-\alpha t^{2}} \leq$ $e^{-\alpha(r / u)^{2}+2 \alpha(r / u) t}$ for $t \geq r / u$. If we choose $\alpha^{-1}=\lambda r$, then all three terms above decay exponentially with distance $r$, and the first term is upper bounded by $c /(\pi u) \max _{0 \leq \tau \leq 1 / u} e^{-r \tau^{2} / \lambda} u^{r} \tau^{r-1}$. Choosing $\lambda$ to maximize the smallest decay coefficient of the three terms, the lower bound for $\xi^{-1}$ is

$$
\xi^{-1} \geq \begin{cases}\frac{\Delta}{2 u} & \text { if } \Delta \leq u \\ \frac{1}{2} W\left(\frac{\Delta^{2} e}{u^{2}}\right) & \text { if } \Delta>u\end{cases}
$$

where $W(x)$ is the Lambert $W$ function (the product logarithm) defined as the solution to the equation $W e^{W}=x$.

As a first specific example, we apply this bound to the 1D TFIM, and in Fig. 8 we give a comparison between our bound with the previous best bound $\xi^{-1} \geq$ $\max _{\mu>0} \mu\left(1+16 J e^{\mu} / \Delta\right)$ given in Ref. [14], and also with the exact solution $\xi^{-1} \geq|\ln (J / h)|$ given in Refs. [81] and [82]. As we see, in this case our bound is always tighter than the previous bound. This is an especially strong improvement in the limit $J \gg h$ where the previous bound gives a constant, while the bound in Eq. (103) gives $\xi \propto$ $\log (J / h)$, in agreement with the exact solution.

As a second example, we apply the bound Eq. (103) to the large- $S$ Heisenberg $X X Z$ model in 1D. Our bound for the LR speed in Eq. (63) gives $u=v \leq 2 X_{1}(J+$ $\left.\sqrt{J^{2}+8 J_{z} J}\right)$, where $J_{x}=J_{y}=J$. Therefore, our bound for $\xi$ remains finite in the limit $S \rightarrow \infty$ provided that the spectral gap $\Delta$ is finite, while the bound given by the previous method [14] $\xi \leq$ (const.) $\times S / \Delta$ diverges linearly in $S$.
Finally, let us discuss the implication of Theorem 1 from Sec. VIE together with the bound in Eq. (103), in the case of perturbed exactly solvable topologically ordered models with a finite local Hilbert space dimension, such as Kitaev's toric code model [61], string-net models [62], the X-cube model [79], and Haah's cubic code model [78]. When these exactly solvable commuting Hamiltonians are perturbed with $J \sum_{i} \hat{V}_{i}$, in the limit $J \rightarrow 0$, the spectral gap $\Delta$ stays finite, while Theorem 1 implies that the LR velocity on the commutativity graph has an upper bound $u \leq c^{\prime} J \ll \Delta$. Therefore, Eq. (103) gives the bound

$$
\xi \leq\left[\frac{1}{2} W\left(\frac{\Delta^{2} e}{u^{2}}\right)\right]^{-1} \propto\left[\ln \frac{\Delta}{J}\right]^{-1},
$$

i.e., when the perturbation is turned off $J \rightarrow 0$, the correlation length $\xi$ vanishes at least as fast as $|\ln J|^{-1}$. The previous LR bounds for this family of perturbed exactly solvable models always give a finite LR velocity when $J \rightarrow 0$, so the previous upper bounds on $\xi$ are also finite when $J \rightarrow 0$, qualitatively looser than our bound.

\section{CONCLUSIONS}

We introduce a method to obtain tighter LR bounds by taking into account the details of the Hamiltonian, and show that this method improves LR bounds both qualitatively and quantitatively. The bounds established in this paper have much tighter LR speeds, especially in three broad scenarios: (1) When the number of local degrees of freedom becomes large, for example in the large-spin TFIM and Heisenberg $X Y Z$ model, the $N$-state truncated $\mathrm{BH}$ model, the large- $N$ SU( $N)$ FH model, and Wen's quantum rotor model. (2) When a set of commuting parameters becomes large, for example in the TFIM at large $J$ and the perturbed toric code model. (3) In large spatial dimension, for example in the TFIM and Wen's quantum rotor model. In these limits our LR speeds have qualitatively better asymptotic scaling than the previous best bounds, and in some cases [e.g., Wen's quantum rotor model and the classical limit of spin- $S$ Ising, Heisenberg and $\mathrm{SU}(N)$ $\mathrm{FH}$ ] our bounds are qualitatively tight in the sense that they scale in the same way as the known semiclassical solution. Our bounds also have superexponential decaying tails $e^{-d_{X Y} \ln d_{X Y}}$ as well as a much tighter short time scaling $O\left(t^{d_{X Y}}\right)$, instead of previous bounds' exponential decay $e^{-\mu d_{X Y}}$ and $O(t)$ scaling. As demonstrated in several examples, the large distance and small time asymptotic behaviors of our bounds are often tightest possible.

The method introduced in this paper follows a simple prescription. To apply this method, one has to (1) write the Hamiltonian in a suitable decomposition, ideally in a form where any two terms either commute or anticommute; (2) draw the commutativity graph introduced in Sec. II A and 
write down the differential equation Eq. (10) whose solution gives LR bounds for the corresponding unequal time commutators; (3) solve the differential equation. Depending on one's specific needs, we discuss different methods to do the last step. For applications of the LR bounds in quantitative estimates, one can solve the linear differential equations numerically. In an infinite system with translation invariance, one can write the solution in a Fourier integral form, which can also be computed efficiently, and in this case we derive a simple formula that upper bounds the LR speed. For general systems lacking translation invariance, we derive a bound of the general form $c\left(u|t| / d_{X Y}\right)^{d_{X Y}}$, which is slightly looser than direct numerical solution to the differential equations, but is simple, much tighter than previous bounds, and retains all of the qualitative improvements offered here, namely the small- $t$ behavior, the superexponential decaying tail, and tighter LR speeds. We illustrate our methods in a wide range of examples in Sec. V, where the resulting LR bounds demonstrate significant improvements over the previous best bounds.

Our tightening of the LR bounds may have profound consequences in the study of both equilibrium and dynamical properties of quantum many-body systems where the LR bounds are applied. As a first demonstration, we show how our bound leads to a tighter bound on the ground-state correlation length in gapped systems, which qualitatively improves previous best bounds in the large- $J$ limit of the $1 \mathrm{D}$ TFIM and large- $S$ limit of $1 \mathrm{D}$ spin- $S$ Heisenberg $X X Z$ model, and the asymptotic behavior of our bound agrees with the exact solution. Our method to obtain much tighter LR speeds will also be useful in the quantum-information context, where efforts have been made to design protocols for faster quantum-state transfer $[7,8]$, or schemes that create certain entangled states [83-85]. Since the LR bounds can be used to bound the time needed for these dynamical processes [3], a sufficiently tight bound not only serves as a criterion to evaluate the performance of a protocol, but also sets a theoretical upper limit on any protocols based on a given physical platform. While previous applications of the LR bounds are mostly limited to analytic proofs, the significant quantitative tightening of the bounds shown in this paper enable numerical applications as well. One such case is to use the LR bound to upper bound the error of a local observable simulated with a finite system. Finally, as we demonstrate at the end of Sec. VID, our bounds are tight enough that they may be used as a rigorous validity check for the various approximation methods used in quantum many-body physics, such as the semiclassical (truncated Wigner) approximation [86-88], mean-field approximations $[89,90]$, and random-phase approximation [91]. An approximation is immediately proved invalid if it predicts a propagation speed $v$ that exceeds our bound, or if it gives ground-state correlation length $\xi$ and spectral gap $\Delta$ that does not satisfy the inequality in Eq. (103).
The methods introduced in this paper may also shed light on some of the important unsolved problems in the area of LR bounds. We mention two of them below.

One important open problem is how to extend LR bounds to systems with an infinite local Hilbert space dimension, such as in systems with interacting bosons. Despite extensive effort, to date there is only some preliminary success in obtaining a finite LR bound in harmonic systems [57,69], or bounds for a restricted set of operators and special initial states [54] in BH-type models. Our method is very promising in this direction, as already demonstrated in this paper, it leads to a finite LR bound for large- $S$ Heisenberg $X Y Z$ model and Wen's quantum rotor model, and shows qualitatively better scaling laws of $v_{\mathrm{LR}}$ with the number of local states in the truncated $\mathrm{BH}$ model and the $\mathrm{SU}(N) \mathrm{FH}$ model, all of which have not been achieved before. In the future, we hope that by extending the methods of this paper, one can find a bigger class of nontrivial interacting models with infinite local Hilbert space whose LR speeds remain finite.

Another open problem is to tighten the LR bounds in systems with power-law decaying interactions [40]. The generalization of our method to this case is straightforward. It may not lead to a qualitative improvement in the most generic case, but when applied to specific models, our method can take advantage of the specific properties of the Hamiltonian to give improved bounds. A promising case is when the long-range interactions are mutually commuting. This includes Coulomb interactions, van der Waals interactions between Rydberg atoms, the $\sigma_{i}^{z} \sigma_{j}^{z}$ interaction in power-law TFIM, all of which are of great theoretical and experimental interests. In Sec. V we see in many examples that our commutativity graph method has a qualitative advantage over previous methods especially when there are lots of commuting terms that have a spatial overlap. It is, therefore, natural to expect that if one combines our commutativity graph method with the current best methods $[36,45,46]$ in treating the long-range part, one can get qualitatively better, model-specific LR bounds in a family of models that are of great physical significance.

\section{ACKNOWLEDGMENTS}

We thank Stefano Chessa, Yichen Huang, Andrew Lucas, Michael Foss-Feig, Putian Li, Bhuvanesh Sundar, and Ian White for discussions. This work is supported by the Welch Foundation (C-1872) and the National Science Foundation (PHY-1848304).

\section{APPENDIX A: A MORE GENERAL METHOD}

In the examples of Sec. V, there are two places where our method slightly deviates from the general methods introduced in Sec. II: in the SU(N) FH model Eqs. (77) and (78) we use a method that is essentially a mixture of the commutativity graph method and the fermion method, 
and in Wen's quantum rotor model Eq. (87) we start with the Heisenberg equation for $\hat{S}_{i j}^{z}$ and $\hat{W}_{i}$ rather than the Hamiltonian terms $\left(\hat{S}_{i j}^{z}\right)^{2}$ and $\hat{W}_{i}$ as we do in Sec. II A. One may, therefore, be curious whether there is a more general prescription behind these seemingly different approaches. Indeed, as we see in this section, the commutativity graph method, the fermion method, the modified fermion method in the $\mathrm{SU}(N) \mathrm{FH}$ model, and the method for Wen's quantum rotor model, are just special cases of a more general method to be introduced below. This general method starts with the Heisenberg equation for an arbitrary set of local operators, thereby allowing a high level of flexibility. The downside of this flexibility is that in some cases one may need considerable physical and mathematical intuition to make the best choice. The general prescription is as follows:

Step 1. Choose a local operator basis $\left\{\hat{\Omega}_{i}\right\}_{i \in M}$ for the Hamiltonian $\hat{H}$, here $M$ is a collection of indices. By basis, we mean that the Hamiltonian can be written as a sum of products of elements of this basis in at least one way (there can be more than one way, in this case we call the basis overcomplete)

$$
\hat{H}=\hat{H}\left[\left\{\hat{\Omega}_{i}\right\}\right]=\sum_{I} h_{I} \prod_{i \in I} \hat{\Omega}_{i},
$$

where $I$ denotes an ordered subset of $M$. For example, in the commutativity graph method, the basis is simply the collection of all individual terms in the Hamiltonian Eq. (4); in the fermion method, the basis is the set of all Majorana operators; in the modified fermion method of FH model, the basis is the set of all Majorana operators and all the $\hat{u}_{j \sigma \sigma^{\prime}}$ terms in Eq. (76), however, since $\hat{u}_{j \sigma \sigma^{\prime}}$ is a quartic product of Majorana operators, this basis is overcomplete; in Wen's quantum rotor model, the basis is the collection of all $\hat{S}_{i j}^{z}$ and $\hat{W}_{i}$. One can easily verify that in all these cases, the Hamiltonian can be written as a polynomial of the basis elements.

Step 2. Write down the Heisenberg equation of motion for the basis operators, as is done in Eqs. (5), (21), (77), (87). For the generic Hamiltonian in Eq. (A1), we have

$$
\begin{aligned}
i \partial_{t} \hat{\Omega}_{i} & =\sum_{I} h_{I}\left[\hat{\Omega}_{i}, \prod_{j \in I} \hat{\Omega}_{j}\right], \\
& =\sum_{J} h_{J}^{\prime} \prod_{l \in J} \hat{\Omega}_{l}, \\
& =P_{i}\left[\left\{\hat{\Omega}_{j}\right\}\right] \hat{\Omega}_{i}+\hat{\Omega}_{i} Q_{i}\left[\left\{\hat{\Omega}_{j}\right\}\right]+R_{i}\left[\left\{\hat{\Omega}_{j}\right\}\right],
\end{aligned}
$$

where in the second line we apply the basic commutation relations of the basis operators to simplify the equation, and in the third line we isolate all the terms that are left proportional or right proportional to $\hat{\Omega}_{i}$, here $P_{i}, Q_{i}, R_{i}$ are some polynomial functions of the basis operators, and $P_{i}\left[\left\{\hat{\Omega}_{j}\right\}\right], Q_{i}\left[\left\{\hat{\Omega}_{j}\right\}\right]$ are assumed to be Hermitian [we can always absorb the non-Hermitian part into $\left.R_{i}\left[\left\{\hat{\Omega}_{j}\right\}\right]\right]$.

Step 3. On both sides of the Heisenberg equation, take the commutator with another local operator $\hat{B}$ (note: if both the lhs and $\hat{B}$ are fermionic operators, anticommutator should be taken). Use Leibniz's rule to expand the product terms, e.g., $[\hat{a} \hat{b} \hat{c}, \hat{B}]=[\hat{a}, \hat{B}] \hat{b} \hat{c}+\hat{a}[\hat{b}, \hat{B}] \hat{c}+\hat{a} \hat{b}[\hat{c}, \hat{B}]$. This is done in Eqs. (6), (22), (88) [and below Eq. (77), implicitly] of the main text. We write the equation in the form

$$
i \partial_{t} \hat{\Omega}_{i}^{B}=P_{i}\left[\left\{\hat{\Omega}_{j}\right\}\right] \hat{\Omega}_{i}^{B}+\hat{\Omega}_{i}^{B} Q_{i}\left[\left\{\hat{\Omega}_{j}\right\}\right]+S_{i}\left[\left\{\hat{\Omega}_{j}\right\},\left\{\hat{\Omega}_{j}^{B}\right\}\right],
$$

where $S_{i}\left[\left\{\hat{\Omega}_{j}\right\},\left\{\hat{\Omega}_{j}^{B}\right\}\right]$ is some polynomial function that is linear in $\hat{\Omega}_{j}^{B}$.

Step 4. Use a change of variable $\hat{\Omega}_{i}^{B}(t) \rightarrow \hat{U}_{i}(t) \hat{X}_{i}^{B}(t)$ $\hat{V}_{i}(t)$ to cancel the first two terms on the rhs of Eq. (A3), where $i \partial_{t} \hat{U}_{i}(t)=P_{i}\left[\left\{\hat{\Omega}_{j}\right\}\right] \hat{U}_{i}(t), i \partial_{t} \hat{V}_{i}(t)=\hat{V}_{i}(t) Q_{i}\left[\left\{\hat{\Omega}_{j}\right\}\right]$ [92]. Equation (A3) becomes

$$
i \partial_{t} \hat{X}_{i}^{B}=\hat{U}_{i}^{\dagger} S_{i}\left[\left\{\hat{\Omega}_{j}\right\},\left\{\hat{\Omega}_{j}^{B}\right\}\right] \hat{V}_{i}^{\dagger} .
$$

Notice also $\left\|\hat{\Omega}_{i}^{B}(t)\right\|=\left\|\hat{X}_{i}^{B}(t)\right\|$ due to $\hat{U}_{i}, \hat{V}_{i}$ being unitary. This is done in Eq. (7) [and below Eq. (77), implicitly] and between Eqs. (88) and (89).

Step 5. Take operator norm on both sides of the resulting operator evolution Eq. (A4), and apply the triangle inequalities on the rhs to get $\left\|S_{i}\left[\left\{\hat{\Omega}_{j}\right\},\left\{\hat{\Omega}_{j}^{B}\right\}\right]\right\| \leq$ $\sum_{j} S_{i j}\left\|\hat{\Omega}_{j}^{B}\right\|$ for suitable nonnegative coefficients $S_{i j}$. Then using the fundamental theorem of calculus and Grönwall's inequality, one can prove that $\left\|\hat{\Omega}_{i}^{B}(t)\right\|$ is upper bounded by the solution to the system of linear differential equations

$$
\partial_{t} \bar{\Omega}_{i}^{B}=\sum_{j} S_{i j} \bar{\Omega}_{j}^{B}
$$

with initial condition $\bar{\Omega}_{i}^{B}(0)=\left\|\hat{\Omega}_{i}^{B}(0)\right\|$. This is done in Eqs. (8)-(10), (23)-(24), (78), (89).

Step 6. Solve the resulting linear differential Eqs. (A5), and calculate $v_{\mathrm{LR}}$ from its maximal eigenfrequency using Eq. (31).

The main flexibility allowed by this general method is the choice of basis operators $\left\{\hat{\Omega}_{i}\right\}_{i \in M}$. A different choice of basis typically leads to a different LR speed. We hope to find a basis that makes the resulting LR speed as small as possible to make the LR bound tighter. We already demonstrate in the main text that the basis $\left\{\hat{\gamma}_{i}\right\}$ used in the minimal Clifford decomposition Eq. (4) satisfying Eq. (18) is a good choice of basis for a large class of spin and interacting fermion models, and the Majorana operator basis $\left\{\hat{c}_{i}\right\}$ satisfying Eq. (20) is good for weakly interacting fermions, in 
the sense that the resulting LR speeds vastly improves the previous results, and the LR bounds typically have tight large distance and small time exponents. We did encounter a few exceptions where other choices are favorable. For example in Wen's quantum rotor model we use the basis $\left\{\hat{S}_{i j}^{z}, \hat{W}_{i}, \hat{W}_{i}^{\dagger}\right\}$, and in the large- $N \mathrm{SU}(N)$ FH model we use $\left\{\hat{c}_{i \sigma}, \hat{u}_{j \sigma \sigma^{\prime}}\right\}$. In addition, in the spin- $S$ Heisenberg model, an alternative, simpler choice of basis $\left\{\hat{S}_{i}^{x}, \hat{S}_{i}^{y}, \hat{S}_{i}^{z}\right\}$ leads to $v \leq 4 d J_{m} X_{1}$, which is the same as Eq. (63) in the $S \rightarrow \infty$ limit. It may be a good future direction to further explore the role of basis operators and hopefully obtain a general prescription on how to find the best choice of basis in a specific model.

[1] E. H. Lieb and D. W. Robinson, The finite group velocity of quantum spin systems, Commun. Math. Phys. 28, 251 (1972).

[2] M. B. Hastings, Locality in quantum systems, arXiv:1008. 5137 (2010).

[3] S. Bravyi, M. B. Hastings, and F. Verstraete, Lieb-Robinson Bounds and the Generation of Correlations and Topological Quantum Order, Phys. Rev. Lett. 97, 050401 (2006).

[4] J. Eisert and T. J. Osborne, General Entanglement Scaling Laws from Time Evolution, Phys. Rev. Lett. 97, 150404 (2006).

[5] K. R. A. Hazzard, M. van den Worm, M. Foss-Feig, S. R. Manmana, E. G. Dalla Torre, T. Pfau, M. Kastner, and A. M. Rey, Quantum correlations and entanglement in farfrom-equilibrium spin systems, Phys. Rev. A 90, 063622 (2014).

[6] C. Gogolin and J. Eisert, Equilibration, thermalisation, and the emergence of statistical mechanics in closed quantum systems, Rep. Prog. Phys. 79, 056001 (2016).

[7] S. Bose, Quantum communication through spin chain dynamics: An introductory overview, Contemp. Phys. 48, 13 (2007).

[8] J. M. Epstein and K. B. Whaley, Quantum peed limits for quantum-information-processing tasks, Phys. Rev. A. 95, 042314 (2017).

[9] P. Calabrese and J. Cardy, Time Dependence of Correlation Functions Following a Quantum Quench, Phys. Rev. Lett. 96, 136801 (2006).

[10] M. Rigol, V. Dunjko, V. Yurovsky, and M. Olshanii, Relaxation in a Completely Integrable Many-Body Quantum System: An Ab Initio Study of the Dynamics of the Highly Excited States of 1D Lattice Hard-Core Bosons, Phys. Rev. Lett. 98, 050405 (2007).

[11] Z.-X. Gong and L.-M. Duan, Prethermalization and dynamic phase transition in an isolated trapped ion spin chain, New J. Phys. 15, 113051 (2013).

[12] J. Eisert, M. Friesdorf, and C. Gogolin, Quantum manybody systems out of equilibrium, Nat. Phys. 11, 124 (2015).

[13] M. B. Hastings, Decay of Correlations in Fermi Systems at Nonzero Temperature, Phys. Rev. Lett. 93, 126402 (2004).
[14] M. B. Hastings and T. Koma, Spectral gap and exponential decay of correlations, Commun. Math. Phys. 265, 781 (2006).

[15] B. Nachtergaele and R. Sims, Lieb-Robinson bounds and the exponential clustering theorem, Commun. Math. Phys. 265, 119 (2006).

[16] M. Cramer and J. Eisert, Correlations, spectral gap and entanglement in harmonic quantum systems on generic lattices, New J. Phys. 8, 71 (2006).

[17] M. B. Hastings, Solving gapped Hamiltonians locally, Phys. Rev. B 73, 085115 (2006).

[18] J. Haegeman, S. Michalakis, B. Nachtergaele, T. J. Osborne, N. Schuch, and F. Verstraete, Elementary Excitations in Gapped Quantum Spin Systems, Phys. Rev. Lett. 111, 080401 (2013).

[19] M. Kliesch, C. Gogolin, M. J. Kastoryano, A. Riera, and J. Eisert, Locality of Temperature, Phys. Rev. X 4, 031019 (2014).

[20] E. Lieb, T. Schultz, and D. Mattis, Two soluble models of an antiferromagnetic chain, Ann. Phys. 16, 407 (1961).

[21] M. B. Hastings, Lieb-Schultz-Mattis in higher dimensions, Phys. Rev. B 69, 104431 (2004).

[22] B. Nachtergaele and R. Sims, A multi-dimensional LiebSchultz-Mattis theorem, Commun. Math. Phys. 276, 437 (2007).

[23] M. B. Hastings, Entropy and entanglement in quantum ground states, Phys. Rev. B 76, 035114 (2007).

[24] M. B. Hastings, An area law for one-dimensional quantum systems, J. Stat. Mech.: Theory Exp. 2007, P08024 (2007).

[25] J. Eisert, M. Cramer, and M. B. Plenio, Colloquium: Area laws for the entanglement entropy, Rev. Mod. Phys. 82, 277 (2010).

[26] M. B. Hastings and X.-G. Wen, Quasiadiabatic continuation of quantum states: The stability of topological ground-state degeneracy and emergent gauge invariance, Phys. Rev. B 72, 045141 (2005).

[27] S. Bravyi, M. B. Hastings, and S. Michalakis, Topological quantum order: Stability under local perturbations, J. Math. Phys. 51, 093512 (2010).

[28] S. Bravyi and M. B. Hastings, A short proof of stability of topological order under local perturbations, Commun. Math. Phys. 307, 609 (2011).

[29] S. Michalakis and J. P. Zwolak, Stability of frustration-free hamiltonians, Commun. Math. Phys. 322, 277 (2013).

[30] M. B. Hastings and S. Michalakis, Quantization of Hall conductance for interacting electrons on a torus, Commun. Math. Phys. 334, 433 (2015).

[31] T. J. Osborne, Efficient Approximation of the Dynamics of One-Dimensional Quantum Spin Systems, Phys. Rev. Lett. 97, 157202 (2006).

[32] T. J. Osborne, Simulating adiabatic evolution of gapped spin systems, Phys. Rev. A 75, 032321 (2007).

[33] T. J. Osborne, Ground state of a class of noncritical onedimensional quantum spin systems can be approximated efficiently, Phys. Rev. A 75, 042306 (2007).

[34] M. P. Woods, M. Cramer, and M. B. Plenio, Simulating Bosonic Baths with Error Bars, Phys. Rev. Lett. 115, 130401 (2015).

[35] M. P. Woods and M. B. Plenio, Dynamical error bounds for continuum discretisation via Gauss quadrature rules-a 
Lieb-Robinson bound approach, J. Math. Phys. 57, 022105 (2016).

[36] M. C. Tran, A. Y. Guo, Y. Su, J. R. Garrison, Z. Eldredge, M. Foss-Feig, A. M. Childs, and A. V. Gorshkov, Locality and Digital Quantum Simulation of Power-Law Interactions, Phys. Rev. X 9, 031006 (2019).

[37] J. Eisert, M. van den Worm, S. R. Manmana, and M. Kastner, Breakdown of Quasilocality in Long-Range Quantum Lattice Models, Phys. Rev. Lett. 111, 260401 (2013).

[38] P. Richerme, Z.-X.n Gong, A. Lee, C. Senko, J. Smith, M. Foss-Feig, S. Michalakis, A. V. Gorshkov, and C. Monroe, Non-local propagation of correlations in quantum systems with long-range interactions, Nature 511, 198 (2014).

[39] Z.-X. Gong, M. Foss-Feig, S. Michalakis, and A. V. Gorshkov, Persistence of Locality in Systems with Power-Law Interactions, Phys. Rev. Lett. 113, 030602 (2014).

[40] M. Foss-Feig, Z.-X. Gong, C. W. Clark, and A. V. Gorshkov, Nearly Linear Light Cones in Long-Range Interacting Quantum Systems, Phys. Rev. Lett. 114, 157201 (2015).

[41] M. F. Maghrebi, Z.-X. Gong, M. Foss-Feig, and A. V. Gorshkov, Causality and quantum criticality in long-range lattice models, Phys. Rev. B 93, 125128 (2016).

[42] T. Matsuta, T. Koma, and S. Nakamura, Improving the Lieb-Robinson bound for long-range interactions, Ann. Henri Poincaré 18, 519 (2017).

[43] D. V. Else, F. Machado, C. Nayak, and N. Y. Yao, Improved Lieb-Robinson bound for many-body hamiltonians with power-law interactions, Phys. Rev. A 101, 022333 (2020).

[44] A. Y. Guo, M. C. Tran, A. M. Childs, A. V. Gorshkov, and Z.-X. Gong, Signaling and scrambling with strongly longrange interactions, Phys. Rev. A 102, 010401 (2020).

[45] C.-F. Chen and A. Lucas, Finite Speed of Quantum Scrambling with Long Range Interactions, Phys. Rev. Lett. 123, 250605 (2019).

[46] T. Kuwahara and K. Saito, Strictly Linear Light Cones in Long-Range Interacting Systems of Arbitrary Dimensions, Phys. Rev. X 10, 031010 (2020).

[47] M. C. Tran, J. R. Garrison, Z.-X. Gong, and A. V. Gorshkov, Lieb-Robinson bounds on $n$-partite connected correlation functions, Phys. Rev. A 96, 052334 (2017).

[48] M. B. Hastings, Locality in Quantum and Markov Dynamics on Lattices and Networks, Phys. Rev. Lett. 93, 140402 (2004).

[49] D. Poulin, Lieb-Robinson Bound and Locality for General Markovian Quantum Dynamics, Phys. Rev. Lett. 104, 190401 (2010).

[50] R. Sweke, J. Eisert, and M. Kastner, LiebRobinson bounds for open quantum systems with longranged interactions, J. Phys. A 52, 424003 (2019).

[51] M. Cheneau, P. Barmettler, D. Poletti, M. Endres, P. Schauß, T. Fukuhara, C. Gross, I. Bloch, C. Kollath, and $\mathrm{S}$. Kuhr, Light-cone-like spreading of correlations in a quantum many-body system, Nature 481, 484 (2012).

[52] P. Jurcevic, B. P. Lanyon, P. Hauke, C. Hempel, P. Zoller, R. Blatt, and C. F. Roos, Quasiparticle engineering and entanglement propagation in a quantum many-body system, Nature 511, 202 (2014).

[53] A. Hamma, F. Markopoulou, I. Prémont-Schwarz, and S. Severini, Lieb-Robinson Bounds and the Speed of Light from Topological Order, Phys. Rev. Lett. 102, 017204 (2009).

[54] N. Schuch, S. K. Harrison, T. J. Osborne, and J. Eisert, Information propagation for interacting-particle systems, Phys. Rev. A 84, 032309 (2011).

[55] D. A. Roberts and B. Swingle, Lieb-Robinson Bound and the Butterfly Effect in Quantum Field Theories, Phys. Rev. Lett. 117, 091602 (2016).

[56] C. K. Burrell and T. J. Osborne, Bounds on the Speed of Information Propagation in Disordered Quantum Spin Chains, Phys. Rev. Lett. 99, 167201 (2007).

[57] M. Cramer, A. Serafini, and J. Eisert, Locality of dynamics in general harmonic quantum systems, arXiv:0803.0890 (2008).

[58] C. K. Burrell, J. Eisert, and T. J. Osborne, Information propagation through quantum chains with fluctuating disorder, Phys. Rev. A 80, 052319 (2009).

[59] D. Damanik, M. Lemm, M. Lukic, and W. Yessen, New Anomalous Lieb-Robinson Bounds in Quasiperiodic XY Chains, Phys. Rev. Lett. 113, 127202 (2014).

[60] Z. Gong, N. Kura, M. Sato, and M. Ueda, Lieb-Robinson bounds on entanglement gaps from symmetry-protected topology, arXiv:1904.12464 (2019).

[61] A. Yu. Kitaev, Fault-tolerant quantum computation by anyons, Ann. Phys. 303, 2 (2003).

[62] M. A. Levin and X.-G. Wen, String-net condensation: A physical mechanism for topological phases, Phys. Rev. B 71, 045110 (2005).

[63] J. Chandra and P. W. Davis, Linear generalizations of Grönwall's inequality, Proc. Am. Math. Soc. 60, 157 (1976).

[64] Z. Nussinov and G. Ortiz, Bond algebras and exact solvability of Hamiltonians: Spin $s=1 / 2$ multilayer systems, Phys. Rev. B 79, 214440 (2009).

[65] E. Cobanera, G. Ortiz, and Z. Nussinov, The bond-algebraic approach to dualities, Adv. Phys. 60, 679 (2011).

[66] References $[3,77,93,94]$ also derive LR bounds that decay superexponentially in distance, and have small- $t$ exponents proportional to the Manhattan distance in real space. But these bounds are still looser than ours in most cases of TFIM. For example, their small- $t$ exponent is $|\vec{r}|$ while our bound in Eq. (51) has small-t exponent $2|\vec{r}|$, and at large $r \equiv$ $|\vec{r}|$, their bound decays like $e^{-r \ln r}$ while our bound decays like $e^{-2 r \ln r}$, due to the fact that the distance on the commutativity graph of TFIM is twice the distance on real space. Also, Ref. [93] only gives the $1 \mathrm{D}$ result and the method is hard to generalize to higher dimensions, while the higherdimensional cases of Refs. [3] and [77] require solving a hard combinatorical problem of summing over paths, the computational difficulty of which may grow exponentially with system size.

[67] The reason for this is that most of the previous LR bounds are very generic to include a large family of models. As we see at the end of Sec. VD, the speed of $d$-dimensional free fermions grows linearly at large $d$, so $v \leq$ (const.) $\times d$ is the best thing a generic bound can do.

[68] F. Duncan M. Haldane, Nonlinear Field Theory of LargeSpin Heisenberg Antiferromagnets: Semiclassically Quantized Solitons of the One-Dimensional Easy-Axis Néel State, Phys. Rev. Lett. 50, 1153 (1983). 
[69] B. Nachtergaele, H. Raz, B. Schlein, and R. Sims, Liebrobinson bounds for harmonic and anharmonic lattice systems, Commun. Math. Phys. 286, 1073 (2009).

[70] J. Eisert and D. Gross, Supersonic Quantum Communication, Phys. Rev. Lett. 102, 240501 (2009).

[71] C. Menotti and N. Trivedi, Spectral weight redistribution in strongly correlated bosons in optical lattices, Phys. Rev. B 77, 235120 (2008).

[72] X.-G. Wen, Artificial light and quantum order in systems of screened dipoles, Phys. Rev. B 68, 115413 (2003).

[73] X.-G. Wen, Quantum order from string-net condensations and the origin of light and massless fermions, Phys. Rev. D 68, 065003 (2003).

[74] M. Levin and X.-G. Wen, Quantum ether: Photons and electrons from a rotor model, Phys. Rev. B 73, 035122 (2006).

[75] Notice that the semiclassical result uses Cartesian distance instead of graph theoretical distance. If we use Cartesian distance as well, then the LR speed in all directions is upper bounded by $2 \sqrt{g J} \min _{\kappa>0} \sqrt{3+\cosh \kappa} / \kappa \approx$ $2.396 \sqrt{g J}$, which is only a factor of 1.69 larger than semiclassical result.

[76] Notice, however, that if the methods of this section are used, then the large- $x$ and small- $t$ exponents of the resulting bounds would not be tight anymore. For example, in the TFIM, both the large- $J$ bound and the large- $h$ bound gives small- $t$ exponent $\eta(\vec{r})=|\vec{r}|$, which is looser than the bound in Sec. V A and exact result by a factor of 2 . Since a tighter LR speed is more important in typical applications, it is generally worthwhile to tighten the LR speed at the cost of compromising the large- $x$ and small- $t$ asymptotic behaviors.

[77] C.-F. Chen and A. Lucas, Operator growth bounds from graph theory, arXiv:1905.03682 (2019).

[78] J. Haah, Local stabilizer codes in three dimensions without string logical operators, Phys. Rev. A 83, 042330 (2011).

[79] S. Vijay, J. Haah, and L. Fu, Fracton topological order, generalized lattice gauge theory, and duality, Phys. Rev. B 94, 235157 (2016).

[80] R. M. Nandkishore and M. Hermele, Fractons, Annu. Rev. Condens. Matter Phys. 10, 295 (2019).

[81] P. Pfeuty and R. J. Elliott, The Ising model with a transverse field. II. Ground state properties, J. Phys. C 4, 2370 (1971).

[82] Theorem 2 of Ref. [2] upper bounds the ground-state correlation by $c_{1} \exp \left(r \Delta / 2 v_{\mathrm{LR}}\right)+c_{2} g(r)$, where $c_{1}, c_{2}$ are some positive constants, and $g(r)$ first appeared in Eq. (30) of the same paper, which, as Ref. [2] claims: "decays faster than exponential in finite range interacting systems." If this claim were correct, one would have the bound $\xi^{-1} \geq$ $\Delta / 2 v_{\mathrm{LR}}$, which is even tighter than our bound in Eq. (103) at large $\Delta / v_{\mathrm{LR}}$. But this bound is actually incorrect. It is true that in locally interacting systems, the LR bound $C_{B}(X, t)$ decays faster than exponential at fixed time, as demonstrated in our paper. However, when the bound is written in the form Eq. (30) of Ref. [2], the function $g(r)$ only decays exponentially. This can be easily verified in the free fermion solution Eq. (85) in our paper, by notic- ing that the Bessel function $J_{x}(x / w)$ decays exponentially with $x$ for arbitrary $w>1$, see Ref. [95]. Also, in the case of $1 \mathrm{D}$ TFIM, the bound $\xi^{-1} \geq \Delta / 2 v_{\mathrm{LR}}$ implies that $\xi^{-1}$ grows at least linearly with $h / J$ as $h / J$ becomes large, while the exact solution only shows a logarithmic growth, see Fig. 8.

[83] S. R. Clark, C. Moura Alves, and D. Jaksch, Efficient generation of graph states for quantum computation, New J. Phys. 7, 124 (2005).

[84] T. Monz, P. Schindler, J. T. Barreiro, M. Chwalla, D. Nigg, W. A. Coish, M. Harlander, W. Hänsel, M. Hennrich, and R. Blatt, 14-Qubit Entanglement: Creation and Coherence, Phys. Rev. Lett. 106, 130506 (2011).

[85] Y. Gao, H. Zhou, D. Zou, X. Peng, and J. Du, Preparation of Greenberger-Horne-Zeilinger and $W$ states on a onedimensional Ising chain by global control, Phys. Rev. A 87, 032335 (2013).

[86] A. Sinatra, C. Lobo, and Y. Castin, The truncated Wigner method for Bose-condensed gases: Limits of validity and applications, J. Phys. B 35, 3599 (2002).

[87] A. Polkovnikov, Phase space representation of quantum dynamics, Ann. Phys. 325, 1790 (2010).

[88] S. M. Davidson, D. Sels, and A. Polkovnikov, Semiclassical approach to dynamics of interacting fermions, Ann. Phys. 384, 128 (2017).

[89] A. Altland and B. D. Simons, Condensed Matter Field Theory (Cambridge University Press, New York, 2010).

[90] A. Kamenev, Field Theory of Non-Equilibrium Systems (Cambridge University Press, New York, 2011).

[91] J. W. Negele, Quantum Many-Particle Systems (CRC Press, Boca Raton, Florida, 2018).

[92] One can actually get rid of more general terms of the form $\sum_{a} \hat{P}_{a} \hat{\Omega}_{i}^{B} \hat{Q}_{a}$ for arbitrary Hermitian operators $\hat{P}_{a}, \hat{Q}_{a}$ (which are implicitly functions of the basis operators $\left\{\hat{\Omega}_{j}\right\}$ ) on the rhs of Eq. (A3), i.e., on the rhs of Heisenberg equation for $i \partial_{t} \hat{\Omega}_{i}^{B}$ one can get rid of any product term that is proportional to $\hat{\Omega}_{i}^{B}$ with coefficients being Hermitian operators. This can be done by going to the Liouville picture, where Eq. (A3) can be rewritten as $\left.\left.i \partial_{t} \mid \Omega_{i}^{B}\right)=\sum_{a} \hat{\Pi}_{a} \mid \Omega_{i}^{B}\right)+\sum_{j \neq i} \hat{S}_{j} \mid \Omega_{j}^{B}$ ), where the operator $\hat{\Omega}_{j}^{B}$ is mapped to a state $\left.\mid \Omega_{i}^{B}\right), \hat{\Pi}_{a}$ denotes the superoperator representing the map $\hat{\Omega}_{i}^{B} \rightarrow \hat{P}_{a} \hat{\Omega}_{i}^{B} \hat{Q}_{a}$, and $\hat{S}_{j}$ denotes the superoperator representing the map $\hat{\Omega}_{j}^{B} \rightarrow S_{i}\left[\left\{\hat{\Omega}_{j}\right\},\left\{\hat{\Omega}_{j}^{B}\right\}\right]$ (remember that $S_{i}\left[\left\{\hat{\Omega}_{j}\right\},\left\{\hat{\Omega}_{j}^{B}\right\}\right]$ is linear in $\hat{\Omega}_{j}^{B}$ ). If $\hat{P}_{a}, \hat{Q}_{a}$ are all Hermitian, then $\hat{\Pi}_{a}$ is Hermitian, and the first term can also be rotated away by a unitary rotation in the Liouville picture, which also preserves the norm of the state $\left.\mid \Omega_{i}^{B}\right)$. Steps 5 and 6 follow without problem.

[93] T. J. Osborne, Simulating evolution of spin systems (2006).

[94] S. Chessa and V. Giovannetti, Time-polynomial LiebRobinson bounds for finite-range spin-network models, Phys. Rev. A 100, 052309 (2019).

[95] G. N. Watson, A Treatise on the Theory of Bessel Functions (Cambridge University Press, Cambridge, UK, 1922). 